\title{
Le développement des métairies dans le comté de Nantes à la fin du Moyen Âge (XIV ${ }^{\mathrm{e}}-\mathrm{XV}^{\mathrm{e}}$ siècles)
}

The development of metayage in the county of Nantes at the end of the Middle Ages ( $14^{\text {th }}-15^{\text {th }}$ centuries)

\section{Brice Rabot}

\section{(2) OpenEdition}

\section{Journals}

Édition électronique

URL : http://journals.openedition.org/abpo/3483

DOI : $10.4000 / a b p o .3483$

ISBN : 978-2-7535-5677-5

ISSN : 2108-6443

\section{Éditeur}

Presses universitaires de Rennes

\section{Édition imprimée}

Date de publication : 30 avril 2017

Pagination : $31-58$

ISBN : 978-2-7535-5675-1

ISSN : 0399-0826

\section{Référence électronique}

Brice Rabot, «Le développement des métairies dans le comté de Nantes à la fin du Moyen Âge (xıve$x v^{e}$ siècles) ", Annales de Bretagne et des Pays de l'Ouest [En ligne], 124-1 | 2017, mis en ligne le 30 avril 2019, consulté le 02 janvier 2020. URL : http://journals.openedition.org/abpo/3483; DOI : 10.4000/ abpo.3483 


\title{
Le développement des métairies dans le comté de Nantes à la fin du Moyen Âge (XIV $-\mathrm{XV}^{\mathrm{e}}$ siècles)
}

\author{
Brice RABOT \\ Docteur en histoire médiévale, chercheur associé au Centre de Recherches \\ en Histoire Internationale et Atlantique (CRHIA) - Université de Nantes
}

Les $\mathrm{XIV}^{\mathrm{e}}$ et $\mathrm{XV}^{\mathrm{e}}$ siècles sont pour les campagnes de l'Ouest français un temps d'épreuves et de profonds bouleversements ${ }^{1}$. Les destructions liées aux guerres, endémiques à la fin du Moyen Âge $^{2}$ - la guerre de succession de Bretagne (1341-1365) est suivie d'affrontements avec la France dans la seconde moitié du $\mathrm{XV}^{\mathrm{e}}$ siècle qui mettent fin à l'autonomie ducale bretonne, les pressions extérieures et l'insécurité ${ }^{3}$, en particulier dans les zones de

1. RАвот, Brice, "Crises et recompositions des campagnes bretonnes méridionales (pays vannetais et nantais) aux xIve et Xve siècles ", dans Josserand, Philippe, LAGET Frédérique et RАВот, Brice, Entre terre et mer. Les campagnes et les sociétés rétro-littorales de l'Ouest atlantique. Mélanges offerts à Jean-Luc Sarrazin (à paraître). Pour une remise en perspective plus large : DuBy Georges et WaLlon, Henri (dir.), Histoire de la France rurale, t. 2 : L'âge classique des paysans de 1340 à 1789, Paris, Seuil, 1975, p. 15-16, 35-39, 77-82; DuBY, Georges, L'économie rurale et la vie des campagnes dans l'Occident médiéval (France, Angleterre, Empire, $I^{e}-X V^{e}$ siècles). Essai de synthèse et perspectives de recherche, vol. 2, Paris, Champs-Flammarion, rééd. 1997 (1 ${ }^{\text {re }}$ éd. : 1962), p. 200-201, 205, 217, 221, 224-225, 228 .

2. LEGUAY, Jean-Pierre et MARTin, Hervé, Fastes et malheurs de la Bretagne ducale (12131532), Rennes, Ouest-France Université, 1997, p. 98-119, 155 (carte des dévastations et des ruines causées par la guerre de succession de Bretagne aux établissements religieux dans les diocèses de Quimper et de Léon, qui laissent entrevoir l'ampleur de la crise), 194-209; RАВот, Brice, Les structures seigneuriales en Bretagne méridionale (pays vannetais et comté de Nantes) à la fin du Moyen Âge (XIV'-début du XVI siècle), thèse de doctorat (dactyl.), Université de Nantes, 2014, p. 331-364, 457-481.

3. À partir de 1403, une série de raids maritimes anglais et bretons ravage les côtes des uns et des autres. La géographie très découpée des littoraux offre autant de sites d'abri pour les pirates et constitue un atout pour les fléaux de la guerre (KNOWLSON, George A., Jean V, duc de Bretagne et l'Angleterre (1399-1442), Rennes, Librairie de Bretagne, 1964, p. 41-47). Des brigands parcourent aussi à l'occasion les campagnes bretonnes, profitant des désordres induits par la guerre. Des bandes pillardes sont ainsi mentionnées dans les environs de Nantes au printemps 1468, lors des opérations militaires françaises dans la région d'Ancenis (Cintré, René, Les marches de Bretagne au Moyen Âge. Économie, 
marches en contact avec les provinces françaises ${ }^{4}$, les désordres économiques et monétaires qui s'accentuent ${ }^{5}$, la paupérisation croissante des populations rurales bretonnes à la fin de la période ${ }^{6}$ ou encore les épisodes météorologiques particulièrement violents dans les décennies 1450-14807 sont autant d'éléments qui mettent à mal les fragiles équilibres du monde rural. Les structures agraires, sur lesquelles pèse une grande part des redevances exigées par les seigneurs et les sieurs ${ }^{8}$, figurent parmi les premières victimes de cette conjoncture instable. Des recompositions de plus en plus nettes s'opèrent, qui conduisent, tout au long du $\mathrm{XV}^{\mathrm{e}}$ siècle, à renforcer le binôme manoir-métairie(s) au détriment des censives ${ }^{9}$, avec des variantes

guerre et société en pays de frontière (XIVe-XV siècles), Pornichet, éd. Jean-Marie Pierre, 1992, p. 142).

4. La période 1470-1483 est ainsi difficile d'un point de vue économique, avant que la situation ne se dégrade encore plus nettement entre 1487 et 1491 (Arch. dép. de Loire-Atlantique, B 13, fo 133, 140 et 141 vo ; Ibid., H 487; LE MEnÉ, Michel, Les campagnes angevines à la fin du Moyen Âge (1350-1530). Étude économique, Nantes, Cid éditions, 1982, p. 253-269, 502-503; Idem, "La conjoncture économique angevine sous le règne de Louis XI ", dans Chevalier, Bernard, Contamine, Philippe (dir.), La France de la fin du $X V^{e}$ siècle : renouveau et apogée. Économie-pouvoir-arts. Culture et conscience nationales, Paris, CNRS, 1985, p. 51-60).

5. KERHERVÉ, Jean, L'État breton aux XIV et XV siècles. Les ducs, l'argent et les hommes, t. 1, Paris, Maloine, 1987, p. 205-206; TOUCHARD, Henri, Le commerce maritime breton à la fin du Moyen Âge, Paris, Les Belles Lettres, 1967, p. 159-162; Idem, dans DeluMEAU, Jean (dir.), Histoire de la Bretagne, p. 190-191; COATIVy, Yves, La monnaie des ducs de Bretagne de l'an mil à 1499, Rennes, PUR, 2006, p 319-338, 370-372, 392-393.

6. Leguay, Jean-Pierre et MARTin, Hervé, Fastes et malheurs..., op. cit., p. 199-207.

7. RAвот, Brice, Les structures seigneuriales..., op. cit., p. 374-379; ATHIMON, Emmanuelle, Les dérèglements du temps et leurs impacts en Anjou, Poitou et Bretagne méridionale, début $X I V^{e}$ siècle-début $X V I^{e}$ siècle, mémoire de master 2 recherche (dactyl.), Université de Nantes, 2013, p. 79, 83-86, 94 .

8. À la suite de Jean Gallet, nous distinguerons les seigneuries des sieuries. Ces dernières peuvent être définies comme des patrimoines fonciers sans juridiction, ce qui les différencie des seigneuries (GALLET, Jean, La Seigneurie bretonne (1450-1680) : l'exemple vannetais, Paris, Publications de la Sorbonne, 1983, p. 79-81). Les sieurs sont le plus souvent issus des rangs de la noblesse, ce qui entretient la confusion. Les textes désignent les sieuries par les termes " manoirs", " lieux nobles " ou " hébergements " (LEGUAY, Jean-Pierre et MARTin, Hervé, Fastes et malheurs..., op. cit., p. 212). De manière plus large, sur la géographie seigneuriale en Bretagne au Moyen Âge, nous renvoyons aux travaux de LA BORDERIE, Arthur de, Essai sur la géographie féodale de la Bretagne, Rennes, PlihonHervé, 1889; CORNULIER, Ernest de, Essai sur le dictionnaire de terres et des seigneuries comprises dans l'ancien comté nantais et dans le territoire actuel du département de la Loire Inférieure, Paris, Dumoulin, 1857, p. 135-136. La Bretagne méridionale, comme le reste du duché, est organisée par les petites structures seigneuriales : "Les seigneuries de dimensions restreintes prédominent dans nos campagnes ", cité dans LEGUAY, Jean-Pierre, Les villes bretonnes à la fin du Moyen Âge, 1364-vers 1514-1515, t. 4, thèse de troisième cycle (dactyl.), Université de Bordeaux, 1978, p. 1044 (reprise dans Les villes du duché de Bretagne aux XIV et XV siècles, Paris, Maloine, 1981, p. 1).

9. Les métairies, situées sur la réserve, sont rarement citées dans les aveux ou les dénombrements. Les exploitants de ces métairies font l'objet d'un contrôle plus étroit que les tenanciers de la mouvance. Dans certains cas, un bail stipulant les obligations des preneurs (les métayers) et du seigneur est rédigé. Très peu de ces contrats nous sont malheureusement parvenus pour la fin du Moyen Âge. Les sources disponibles aujourd'hui 
selon les lieux. La fin du Moyen Âge marque un tournant majeur avec la mise en œuvre de structures agraires qui, dans leurs grands traits, vont perdurer et se consolider au cours de l'époque moderne, jusqu'à la Révolution au moins ${ }^{10}$.

Les enjeux sont, on le voit, multiples. Les étudier tous impliquerait de mener l'analyse dans le cadre d'un espace beaucoup plus large que celui qui est traité ici. Pour mener à bien l'examen tout en cherchant à dégager quelques lignes de force, nous avons choisi de nous concentrer sur un espace suffisamment ample et cohérent, tant d'un point de vue des structures agraires que de la documentation, celui du comté de Nantes. Enserré entre le Poitou au sud, l'Anjou à l'est, l'Atlantique à l'ouest, la Vilaine et le pays de Châteaubriant au nord, le comté de Nantes permet de confronter des sources qui ne sont pas limitées à un seul terroir mais qui, au contraire, sont révélatrices de la diversité des situations rurales rencontrées en Bretagne méridionale ${ }^{11}$.

La conservation croissante des aveux, des minus, des dénombrements et des comptes au long des $\mathrm{XIV}^{\mathrm{e}}$ et $\mathrm{XV}^{\mathrm{e}}$ siècles pour un nombre de plus en plus grand de seigneuries/sieuries permet de porter différents regards sur les évolutions que connaissent les structures agraires en s'intéressant aux rythmes, aux lieux et, plus difficilement, aux motivations qui ont conduit les détenteurs de fiefs et autres biens fonciers à privilégier progressivement les métairies pour mettre en valeur leur patrimoine.

Après avoir présenté plus en détail le contexte de cette recherche, en insistant particulièrement sur les sources et leurs apports, un tableau de la dualité censives-métairies sera esquissé avant de remettre en perspective les évolutions dégagées.

\section{Le contexte de la recherche}

\section{Les sources : apports et perspectives}

L'étude des campagnes du comté de Nantes présente une première difficulté majeure qui explique, pour partie, que les historiens l'aient délaissée :

ne permettent guère de préciser le statut juridique des terres baillées en métayage en dehors des études de cas, nécessairement ciblées sur une période et une aire géographique précises. Il n'est donc pas possible, dans cette présentation générale, d'apporter une réponse valable pour l'ensemble de l'espace étudié. Cet article s'inscrit en outre dans la continuité des recherches que nous avons entamées lors de notre thèse de doctorat.

10. Gallet, Jean, Seigneurs et paysans bretons du Moyen Âge à la Révolution, Rennes, Ouest-France Université, 1992, p. 18-53, 116-137. Les paysages gardent encore aujourd'hui la trace, dans leur trame, de ces structures (RENARD, Jean, Les campagnes nantaises : un demi-siècle de révolutions sociales et paysagères, 1960-2010, Rennes, PUR, 2012, p. 47-50).

11. La Bretagne comprend deux espaces majeurs, le littoral et l'arrière-pays, qui présentent des traits spécifiques. Le littoral, l'Armor en breton, est densément peuplé et relativement bien mis en valeur. L'arrière-pays, l'Arcoat, est, quant à lui, dominé par des terres plus difficiles à mettre en valeur et moins densément peuplées. Voir à ce sujet MEYNiER, André, Atlas de la géographie de la Bretagne, Paris, Flammarion, 1976, p. 51-52. 
les sources sont très disparates d'une seigneurie à l'autre, d'une période à l'autre, et aussi souvent trop laconiques pour permettre des conclusions générales. Cet élément doit être souligné pour bien comprendre l'absence de monographie consacrée au métayage et à son développement à la fin du Moyen Âge dans cette région, sur le modèle de ce que Louis Merle a pu réaliser pour la Gâtine poitevine ${ }^{12}$ ou de ce que Michel Le Mené a pu conduire pour l'Anjou aux XIV et $\mathrm{XV}^{\mathrm{e}}$ siècles ${ }^{13}$. Avant d'aller plus loin, il convient donc de présenter les sources disponibles pour les campagnes du comté de Nantes, en soulignant à la fois leurs apports, mais aussi leurs limites et les points qui restent aujourd'hui encore en suspens.

Les aveux représentent de loin la première sorte de source utilisable, du fait de leur importance numérique dans les fonds d'archives ${ }^{14}$ et, dans une moindre mesure, de la diversité des indications qu'ils contiennent. Mais ces aveux sont très inégalement conservés selon les seigneuries et selon les périodes. Deux grandes catégories peuvent être distinguées :

- les aveux pour rachat ${ }^{15}$, c'est-à-dire ceux qui sont établis à la mort du détenteur d'un domaine ou fief en vue d'établir le montant de la redevance à verser au duc de Bretagne - le rachat - pour pouvoir hériter des biens et des droits attachés au domaine ou au fief. S'intéressant en priorité aux biens et aux droits concédés, ces aveux se limitent le plus souvent à citer les métairies, sans apporter d'indications plus précises sur leur étendue, le nombre des exploitants ou encore la composition de l'exploitation ${ }^{16}$.

12. Merle, Louis, La métairie et l'évolution agraire de la Gâtine poitevine de la fin du Moyen Âge à la Révolution, Paris, SEVPEN, 1958, p. 47-96.

13. LE MENÉ, Michel, Les campagnes angevines..., op. cit., p. 188-189, 213, 220-221; SICARD, Germain, Le métayage dans le Midi toulousain à la fin du Moyen Âge, Toulouse, Soubiron, 1956.

14. La série B des Archives départementales de Loire-Atlantique est ainsi constituée quasi-exclusivement d'aveux rendus par les seigneurs ou leurs tenanciers au moment du rachat à la Chambre des comptes des ducs de Bretagne. La sous-série $1 \mathrm{E}$, intitulée "Titres féodaux ", toujours pour les Archives départementales de Loire-Atlantique, regorge également d'aveux, bien que ses fonds n'aient pas été classés ni aussi bien répertoriés que ceux de la série B. Quelques aveux peuvent être retrouvés, au gré des dépouillements, dans les séries $\mathrm{G}$ (clergé séculier), $\mathrm{H}$ (clergé régulier) et J (fonds entrés par voie extraordinaire) dans les Archives départementales de Loire-Atlantique. Pour davantage de détails, nous renvoyons au répertoire présenté dans RABOT, Brice, Les structures seigneuriales..., op. cit., p. 25-55.

15. Les aveux pour rachat sont aussi appelés "minus ". Ils présentent en effet, par le menu, les différentes sources de droits et de revenus seigneuriaux, afin d'établir le montant correspondant du rachat dû au duc de Bretagne.

16. Pour ne citer qu'un exemple : "Confessons et avouons tenir par douaire nous escheu et avenu par le deceix et trespas d'iceluy de Craon le chastel, chastellenie, terre et seignourie dudit lieu de La Benaste, o ses appartenances et appendances quelxconques, tant chastel, chastellenie, droit de guiet, que rentes par deniers, blez, chappons, poulles, ouayes, moutons, blez et vin de rente, prez, vignes, tiers, quars et complans de vignes, foretz, boaies enciens et de revenue, estaige, garaines, moulins, destroit et resoul de molins, droit de juridicion haulte, basse et moyenne, hommes, homaiges, rachaz, soulz rachatz d'espaves, galoiz, droit de quictances, de deserence et succession de bastars, de ventes et des mestaeries, terrages [...] et autres heritages, droiz heritelz et choses 
Les aveux pour rachat sont aussi trop espacés dans le temps pour rendre compte finement du rythme des évolutions. Certains sont distants de plusieurs décennies, sans qu'il soit possible de combler les lacunes, faute de disposer d'autres documents ${ }^{17}$;

- les aveux rendus par les tenanciers à leur seigneur/sieur au moment du rachat ou à la demande du propriétaire foncier jettent un autre éclairage sur les métairies. La plupart ne présentent, là encore, que des traits généraux, relatifs le plus souvent à la liste des sommes dues par les tenanciers. Certaines métairies sont alors incidemment mentionnées ${ }^{18}$, mais trop d'actes restent vagues sur la nature du bien exploité. Les rédacteurs se contentent en effet très souvent d'énoncer des "biens " ou des "terres" sans forcément prendre soin de préciser le régime sous lequel se placent les exploitations en question ${ }^{19}$. Il en résulte une difficulté supplémentaire pour les historiens pour bien saisir les rythmes et les modalités d'évolution des structures agraires.

Le croisement des sources est dans ces conditions particulièrement indispensable. Les comptes, très disparates eux aussi et en nombre limité aujourd'hui ${ }^{20}$, permettent de compléter ces informations. Les aléas de la conservation et les tris opérés par les seigneurs ou leurs agents ont éliminé de nombreux registres ${ }^{21}$. Ceux qui nous sont parvenus restent donc très espacés dans le temps - aucun ne remonte avant les années 1420

heritelles quexlconques [...] ", extrait du minu pour rachat rendu le 17 août 1462 par la dame de La Suze, veuve de Jehan de Craon (Arch. dép. de Loire-Atlantique, B 1816).

17. Pour ne prendre qu'un exemple, parmi tant d'autres, nous citerons la seigneurie de La Hodinière, au Loroux-Bottereau, où quatre aveux nous ont été transmis, datant de 1401, 1447, 1473 et 1478 (Arch. dép. de Loire-Atlantique, B 1842).

18. Par exemple : "Savoir est la moitié de la mectié des quarterons de Corleteau, de La Roupiere, de Joinbus, de la metaerie appartenant audit deffunct Gerart de La Noé, quelx quarterons es tiennent et labourent André de Rectuon, Julien Cariolle, Michel Gaultier et Perrot Moreau et [...]. ", extrait du minu rendu le 12 août 1475 par Jehan Chaboz (Arch. dép. de Loire-Atlantique, B 1816).

19. Pour ne citer qu'un exemple : « [...] Item, sur le quarteron de la Lebraudiere, une myne d'avoyne et ung denier à celuy qui lye le sac. [...] ", extrait de l'aveu rendu le 19 juin 1503 par Pierre Goheau (Arch. dép. de Loire-Atlantique, B 1858). Aucun autre élément ne permet de distinguer la nature des biens en question.

20. Seules les séries comptables d'Ancenis (Arch. dép. de Loire-Atlantique, E 261, E 262, E 263, E 264; 16 cahiers), de Belligné (Idem, E 267, E 268; 14 cahiers), de Varades (Id., E 277; 6 cahiers), des Huguetières (Id., E 500, E 501, E 502, E 503; 22 cahiers), de La Blanchardais (Id., 1 E 221, 1 E 222, 1 E 223; 23 cahiers), de Saffré (Id., 1 E 674; 9 cahiers) nous sont parvenues, avec des lacunes chronologiques plus ou moins prononcées selon les cas. D'autres séries, à l'état de fragments - jamais plus de trois registres -, nous ont été transmises pour le temporel (Id., E 66; un cahier) et la comptabilité de l'évêché de Nantes (Id., G 37; 2 cahiers), le marquisat d'Assérac (Id., E 299; 2 cahiers), le marquisat de Blain (Id., E 329; 2 cahiers), la châtellenie de Machecoul (Id., E 522; 2 cahiers) ou encore la seigneurie d'Anetz (Id., 1 E $333 ; 3$ cahiers).

21. L'incendie du château de Blain en novembre 1793 a par exemple entraîné la perte de l'énorme chartrier des Rohan, que l'on peut aisément qualifier de perte archivistique majeure de par l'importance et la richesse de ses fonds (HALGOUËT, Hervé du, "Le chartrier de Blain ", Annales de Bretagne, t. 35, n 1, 1921, p. 86). Les destructions d'archives financières opérées au moment de la Révolution, notamment, ont elles aussi été sélectives 
pour le comté de Nantes en dehors du domaine ducal et toutes les séries sont incomplètes sur le plan chronologique ${ }^{22}$ - et ne concernent surtout qu'un nombre réduit de seigneuries, de la modeste seigneurie foncière ( $\mathrm{La}$ Blanchardais) à la châtellenie (Saffré, Machecoul, Belligné) ou la prévôté (Ancenis) pour les structures les plus importantes dans la hiérarchie seigneuriale bretonne.

Certains cahiers attestent la présence de métairies et listent quelques redevances tirées de leur exploitation. Les comptes de la prévôté d'Ancenis révèlent ainsi le poids grandissant des revenus de l'élevage, avec les ventes de la laine et des bêtes des trois métairies dans les années 1460-1470 (laine) et 1480-1490 (bêtes) (figures 1 et $2^{23}$ ), au moment où les principales crises politiques et militaires ébranlent les équilibres dans les zones de marches ${ }^{24}$. Ces éléments présentent un premier intérêt. Ils montrent le lien entre le développement des métairies ${ }^{25}$ et le poids grandissant des crises sur les revenus seigneuriaux, ce qui conduit les détenteurs de domaines et de fiefs à réorienter les modes du prélèvement pour tenter de maintenir ou, à défaut, de limiter la diminution des revenus tirés des exploitations agricoles. Les comptes ne permettent toutefois pas à eux seuls de saisir les logiques ou les modalités concrètes du développement des métairies à la fin du $x^{\mathrm{e}}$ siècle car tel n'est pas leur objectif. Les receveurs, qui sont chargés de présenter les registres aux auditeurs à la fin de leur exercice comptable, ne retranscrivent dans les cahiers que les éléments indispensables pour justifier la levée, ou l'absence de levée, des rentes. Les motifs qui conduisent les seigneurs à exiger telle ou telle redevance sont en conséquence passés sous silence; ils sont en effet inutiles pour les receveurs qui n'ont pas à les présenter dans leurs registres.

Les baux, par contre, rendent possible une telle approche. Malheureusement, à la fin du Moyen Âge, ils sont en nombre trop réduit et sont surtout trop circonscrits à des espaces fragmentés pour que soit tenté, pour le comté de Nantes, ce qu'a fait Louis Merle pour la Gâtine poitevine, une mise en perspective générale. Toute étude n'est néanmoins pas impossible. Jean-Luc Sarrazin a ainsi mis en évidence, en partant de l'étude des fonds notariaux légués par Sixt Nevouet, petit notaire rural de

(WISMES, baron de, "Le trésor de la rue des Caves à Nantes ", Revue de Bretagne et de Vendée, t. 5, 1859, p. 152-162, 311-335).

22. C'est le cas de la seigneurie de La Blanchardais (Vue), avec des lacunes entre 1439 et 1448,1475 et 1484, 1490 et 1495, à des moments cruciaux où les structures seigneuriales doivent faire face à de graves crises (Arch. dép. de Loire-Atlantique, 1 E 221 à 223; RABOT, Brice, Les structures seigneuriales..., op. cit., p. 106, 410-417, 446-454, 497-501).

23. Arch. dép. de Loire-Atlantique, E 261 (1 à 5), E 262 (1 à 4), E 263 (1 à 4), E 264 (1).

24. Voir à ce sujet CInTRÉ, René, Les marches de Bretagne au Moyen Âge..., op. cit., p. 141142 ; RABOT, Brice, Les structures seigneuriales..., op. cit., p. 460-480; LEGUAY, Jean-Pierre et MARTIN, Hervé, Fastes et malheurs..., op. cit., p. 199-207.

25. Les métairies de la prévôté d'Ancenis mentionnées dans les registres de compte représentent ainsi 93,1 \% des 137,8 hectares de la réserve seigneuriale (VIGNEAU, Thomas, Les métairies de la prévôté d'Ancenis à la fin du Moyen Âge (vers 1450-1525), mémoire de maîtrise (dactyl.), Université de Nantes, 1994, p. 40). 
Figure 1 - Revenus tirés des ventes de laine des métairies de la prévôté d'Ancenis d'après les indications fournies dans les comptes des receveurs (1463-1501)

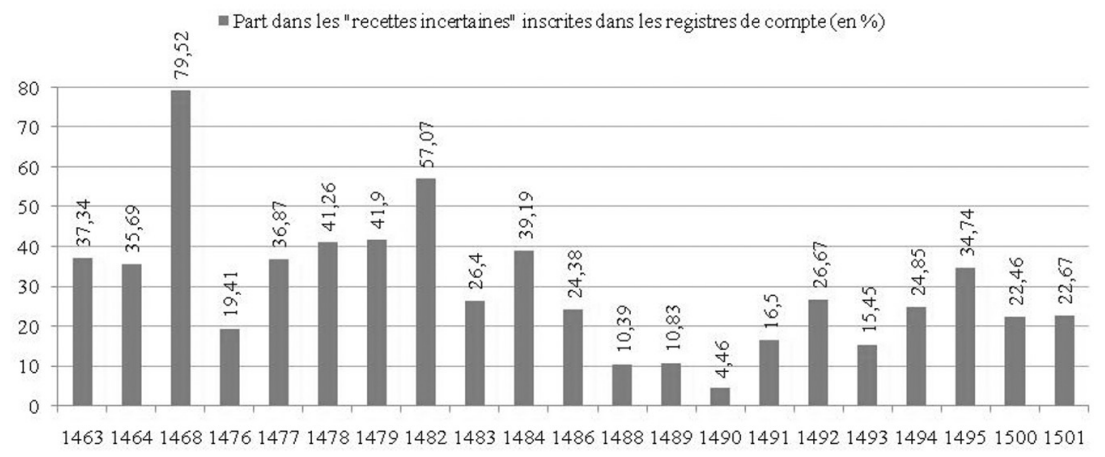

\section{Figure 2 - Revenus tirés des ventes de bêtes des métairies de la prévôté d'Ancenis d'après les indications fournies dans les comptes des receveurs (1463-1501)}

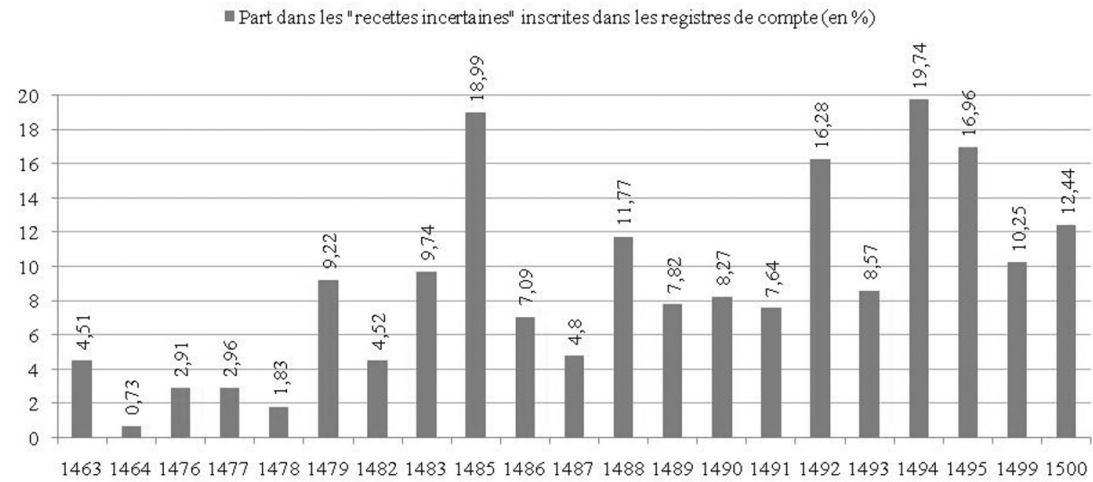

Saint-Philbert-de-Grand-Lieu à la fin du Xve siècle, complétés par les actes transmis par Pierre Chatau, pour la paroisse du Pellerin, les rythmes et les modalités des recompositions agraires qui conduisent à la mise en place des métairies dans ces espaces au cours de la seconde moitié du Xve siècle, au moment où s'opère la reconstruction agraire ${ }^{26}$. Les métairies sont ici de véritables outils de reconstruction et de redressement agraire pour les seigneurs/sieurs, à l'instar de ce que l'on observe en Poitou ou dans le sud

26. SARRAZIN, Jean-Luc, "Les activités d'un rassembleur de terres en Pays de Rais vers le milieu du XV siècle ", Annales de Bretagne, t. 88, nº 2, 1981, p. 135-155. 
de la France. Les rapports d'autorité sont eux aussi redéfinis dans le cadre du métayage. Les seigneurs/sieurs, en imposant certaines obligations aux preneurs ${ }^{27}$, exercent un contrôle beaucoup plus étroit sur leurs exploitants agricoles. Mais de telles études s'avèrent difficiles pour ne pas dire impossibles à conduire ailleurs pour le comté de Nantes, faute de tels documents.

\section{Une position de carrefour}

Une autre originalité tient à la situation géographique du comté de Nantes. Véritable carrefour entre la Bretagne et la France avec l'Anjou à l'est et le Poitou au sud, le comté de Nantes est un territoire ouvert aux échanges et aux influences extérieures. Ce trait est primordial, car il permet de remettre en perspective les observations effectuées dans un cadre plus large que celui de la seule Bretagne méridionale. L'Ouest français connaît à la veille des Temps modernes de profonds bouleversements avec le renforcement du métayage ${ }^{28}$ et le développement de nouveaux réseaux de circulation. Les échanges traversent ainsi le comté de Nantes et irriguent de manière diffuse l'ensemble du territoire. D'aucuns ont d'ailleurs pu parler de " porte " pour souligner cette singularité, à l'instar d'Henri Touchard pour le comté tout entier ${ }^{29}$ ou d'Alain Chauvet analysant le rôle de charnière joué par la ville de Nantes entre les campagnes alentour et le royaume de France ${ }^{30}$. Première ville du comté, bien reliée au reste du comté par la Loire, l'Erdre et la Sèvre nantaise, Nantes fait figure de véritable pivot pour les hommes, les biens et, partant, la diffusion des influences extérieures. La multiplication des péages et autres passages entre Nantes et l'Anjou par exemple, via la Loire, en témoigne ${ }^{31}$. Elle offre en outre aux seigneurs d'autres sources de revenus, parfois lucratives si l'on en juge par les indications transmises dans les aveux ${ }^{32}$, ce qui leur donne ensuite les moyens d'entreprendre des opérations de remembrement.

27. Dans le hameau de Launay par exemple, à Rouans-en-Retz, les exploitants sont tenus de consacrer une somme fixée par contrat - 100 sous - à reconstruire l'hébergement ou à bâtir une maison, le tout dans un délai de 5 à 10 ans (Arch. dép. de Loire-Atlantique, H 49, cité par SARRAZIN, Jean-Luc, op. cit., p. 138).

28. LE MENÉ, Michel, « Les redevances à part de fruits dans l'Ouest de la France au Moyen Âge ", Les revenus de la terre, complant, champart, métayage en Europe occidentale (IX ${ }^{e}$ XVIII ${ }^{e}$ siècles), Flaran, 7, Auch, 1985, p. 9.

29. TOUCHARD, Henri, Le commerce maritime breton..., op. cit., p. 45.

30. Chauvet, Alain, Porte nantaise et isolat choletais : essai de géographie régionale, thèse de doctorat (dactyl.), Université de Nantes, 1986, p. 127-154, 159-209.

31. RАвот, Brice, Les structures seigneuriales..., op. cit., carte $\mathrm{n}^{\circ} 10$, p. 247.

32. Le passage d'Oudon, sur la Loire, rapporte ainsi en moyenne près de 80 livres par an au seigneur : " [...] Item, y a un certain devoir sur Loyre que l'en prent sur les marchandies et denrées ensuivantes. Savoir est sur chacun muy de blé, montent et bessent par la ripviere de Loire, II deniers. Pour chacun muy de vin, II deniers. Pour chacun muy de seil, II deniers. Pour chacun challon portant VI muyz de [...] moins ou portant plus doit un sallage qui vault IX soulz. Pour chacun muy de noez, II deniers. Et de tout autre meage pour chacun muy, II deniers, pour tout challon et vessel qui porte peaultre, quelconque marchandie qu'il portege, pour chacune peaultre, III deniers. Pour le nouage de chacune 
Les autres cours d'eau ne doivent pas non plus être négligés. L'Isar, au nord-ouest du comté, relie l'arrière-pays rural du Gâvre au port de Redon, via la Vilaine, véritable pivot pour le trafic avec la Haute-Bretagne et le pays vannetais $^{33}$. La Sèvre nantaise, au sud-est, permet quant à elle aux seigneurs ruraux du vignoble de rejoindre la ville de Nantes et d'y écouler les parts de vendanges perçues sur les exploitants vers le reste du comté ou vers l'Anjou. Ces échanges ont une incidence certaine sur le choix du prélèvement en numéraire - les paysans doivent alors vendre eux-mêmes leurs productions pour pouvoir ensuite s'acquitter des redevances seigneuriales - ou en nature : les seigneurs, ou leurs agents, revendent sur les marchés le produit des rentes perçues des tenanciers. Prélèvements en numéraire et en nature ne doivent, dans ces conditions, pas être opposés. Ils sont en fait complémentaires ${ }^{34}$, ce qui explique la coexistence de différents types d'exploitations.

La Loire constitue un dernier élément structurant dans la géographie agraire du comté de Nantes. Elle représente en effet une contrainte majeure dans la mise en valeur des terres et le développement des relations entre ses deux rives. Des seigneuries, telle l'abbaye de Buzay, possessionnée à Savenay, à Donges, à Cordemais ou encore à Saint-Étienne-de-Montluc au nord du fleuve, mais implantée à Rouans ${ }^{35}$, en plein cœur du pays de Retz, au sud, doivent mettre en place des stratégies de circulation pour gérer au mieux leur patrimoine tout en respectant les coutumes, qui varient d'un espace à l'autre. Le métayage offre ainsi une solution parmi d'autres, en déléguant une partie de l'exploitation à des preneurs chargés de la faire fructifier, tout en maintenant pour les moines de Buzay, si nous reprenons cet exemple, un droit de regard et un encadrement plus étroit comparé à celui qu'ils exercent sur les censives.

\section{Une zone de marches}

Le contexte frontalier est un dernier point important à souligner. Les zones frontalières - les marches - donnent lieu à des organisations spé-

sentine, II soulz VI deniers, la premiere foiz que elle passe. Pour chacun millier de fer et d'acier, XII deniers. Pour chacune meulle mollin, IIII deniers. Pour chacun millier de merrain et aisil, IIII deniers. Pour chacune cacre de cuir, XVI deniers. Pour chacune charge de estain et de acier, IIII deniers. De chacun cent de lampraes, d'allouses, de saulmons, de peisson seit et de toute paerie de peissons, de harens, de figues de [...] de ouignon et ails, le cinquiesme de chacune espece. Pour chacune couecte de plume, de chacune comere, IIII deniers. Pour chacune huge, de chacun pie, IIII deniers. Et si il trespasse lesdiz acquistz senz poier, ilz doivent prendre les denrées et le vessel. Lequel devoir peut bien valoir communs ans IIIIXX livres [...] " (Arch. dép. de Loire-Atlantique, B 1848 (3), fo 3 r $^{\circ}$ ).

33. Pichot, Daniel et Provost, Georges (dir.), Histoire de Redon. De l'abbaye à la ville, Rennes, PUR, 2015, chap. 8, p. 132-148.

34. RABOT, Brice, Les structures seigneuriales..., op. cit., p. 189-250.

35. SARRAZIN, Jean-Luc, Recueil et catalogue des actes de l'abbaye cistercienne de Buzay en Pays de Rais (1135-1474), thèse de troisième cycle (dactyl.), 4 tomes, Université de Nantes, 1977, p. 112-132. 
cifiques où l'autorité est partagée soit à parts égales, soit au profit des seigneurs bretons ou angevins/poitevins. L'on parle alors, dans ce dernier cas, de marches avantagères ${ }^{36}$. Dans les marches communes, les seigneurs bretons et français disposent en théorie de l'autorité conjointe et équilibrée, sans que l'un ne prenne l'ascendant sur l'autre. Les paroisses de La Remaudière ${ }^{37}$ et de La Boissière-du-Doré, au sud-est du comté, sont ainsi placées sous ce statut (figure 3).

Les travaux anciens d'Émile Chénon ${ }^{38}$ ou ceux, beaucoup plus récents, de René Cintré ${ }^{39}$ ont insisté sur les conséquences économiques et territoriales de cette organisation si particulière. Loin d'être des barrières, empêchant ou bloquant les flux de différentes natures, les marches se trouvent être au contraire des espaces soumis à des échanges plus ou moins larges, plus ou moins légaux également par rapport à la fiscalité, en particulier celle du sel, au cour des parcours. Ces zones contribuent à favoriser les mobilités et les flux qui marquent en retour les structures agraires avec force, comme le laisse entrevoir l'examen plus approfondi des cartes à des époques-charnières comme la fin du XIV et, plus encore, la fin du XVe siècle. Les métairies connaissent à cette dernière période un essor remarquable en Poitou et en Anjou, en lien avec la reconstruction agraire postérieure à la guerre de Cent Ans.

\section{Vers une dualité des structures agraires}

Les emprunts à la géographie s'avèrent particulièrement judicieux et appropriés pour mieux saisir les rythmes et les modalités de développement des métairies à la fin du Moyen Âge. L'emboîtement des échelles permet ainsi d'interroger la complexité des structures agraires observées aux $\mathrm{XIV}^{\mathrm{e}}$ et $\mathrm{XV}^{\mathrm{e}}$ siècles et de les remettre en perspective dans le temps long ${ }^{40}$. Quelques caractéristiques plus précises peuvent dès lors être dégagées en fonction des sous-ensembles régionaux.

36. Pour une présentation plus précise, voir CHÉNON, Émile, "Les Marches séparantes d'Anjou, Bretagne et Poitou ", Nouvelle revue de droit historique français et étranger, t. 16, 1892, p. 18-62, 165-211.

37. La commune de La Remaudière se trouve aujourd'hui placée en Loire-Atlantique, tandis que celle de La Renaudière se situe dans le département du Maine-et-Loire (cette dernière est replacée sur la figure 3).

38. Idem.

39. CinTRÉ, René, La frontière franco-bretonne au Moyen Âge. Économie, mentalités, guerres et sociétés en pays de marches $V^{e}-X V t^{e}$ siècles, thèse de doctorat (dactyl.), Rouen, Rennes, 1989, 3 vol., reprise dans Les marches de Bretagne au Moyen Âge. Économie, guerre et société en pays de frontière (XIV $-X V^{e}$ siècles), Pornichet, éd. Jean-Marie Pierre, 1992, p. 53-63; Id., "Activités économiques dans les marches de Bretagne aux XIV et XV siècles ", Annales de Bretagne et des Pays de l'Ouest, t. 101, no 4, 1994, p. 7-36.

40. PALIERne, Jean-Max, Les forêts et leur environnement dans les pays ligéro-atlantiques nord. Recherches et réflexions biogéographiques sur les discontinuités et la dynamique des paysages naturels et humains, doctorat d'État (dactyl.), Université de Rennes 2, 1975. 


\section{Figure 3 - Marches séparantes entre Bretagne, Anjou et Poitou à la fin du Moyen Âge}

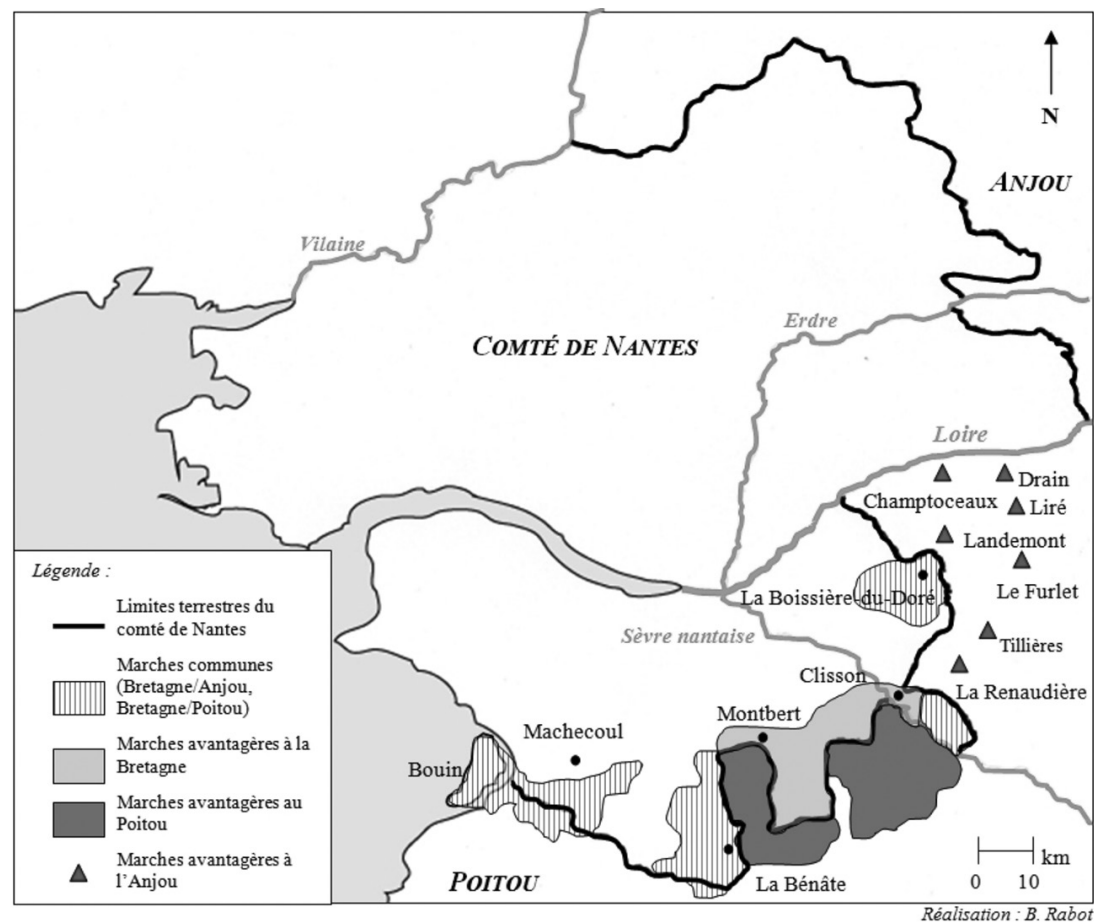

\section{Le maintien des censives au nord de la Loire}

Dans la première moitié du $\mathrm{XV}^{\mathrm{e}}$ siècle, comme au siècle précédent, le maintien des censives y apparaît nettement (figure $4^{41}$ ). La meilleure

41. RАвот, Brice, Les structures seigneuriales..., op. cit., p. 399-437. D'après Arch. dép. de Loire-Atlantique, B 50, B 125, B 126, B 127, B 128, B 129, B 130, B 1441, B 1458, B 1817, B 1819 , B 1825 , B 1827 , B 1829 , B 1831 , B 1842 , B 1843 , B 1846, B 1859 , B 1866 , В 1870 , В 1874, В 1881, В 1885, Е 267, Е 306, Е 336, Е 362, Е 366, E 450, E 458, E 471, E 473, E 485, E 489, E 490, E 495, E 506, Е 508, E 514, E 517, E 536, E 539, E 557, E 600, 1 E 65, 1 E 156, 1 E 176, 1 E 217, 1 E 322, , 1 E 323, 1 E 337, 1 E 338, 1 E 346, 1 E 351, 1 E 674, 1 E 746, G 21, G 37, G 151, G 155, G 226, G 251, G 334, H 3, H 4, H 5, H 7, H 10, H 13, H 14, H 16, H 17, H 34, H 39, H 76, H 515, 217 J 15, 217 J 22; MoRICE, dom Hyacinthe, Mémoires pour servir de preuves à l'histoire ecclésiastique et civile de Bretagne, t. 1, Paris, rééd. 1974 (1 $1^{\text {re }}$ éd. 1742-1746), p. 441; BlanchaRD, René, Lettres et mandements de Jean v, duc de Bretagne, t. 7, 1891, p. 139-140, 141-143; Cartulaire des sires de Rays (1160-1449), publié par BlanCHARD, René, Archives historiques du Poitou, t. 30, Poitiers, 1899, pièce cxLI, p. 116-120, pièce CCCXVII, p. 478-479, pièce CCCXXI, p. 482-483; SADDIER, Évelyne, Une seigneurie rurale du pays nantais au XVe siècle : les Huguetières à travers les comptes du receveur, mémoire de DES (dactyl.), Centre universitaire de Savoie, Chambéry, 1975, p. 7-8. Sont inscrits ici le nombre d'exploitations et de tenanciers/métayers cités dans les aveux et les dénombrements. 
Figure 4 - Répartition spatiale des structures agraires en comté de Nantes d'après les indications des sources issues des fonds des Archives départementales de Loire-Atlantique (années 1400-1440)

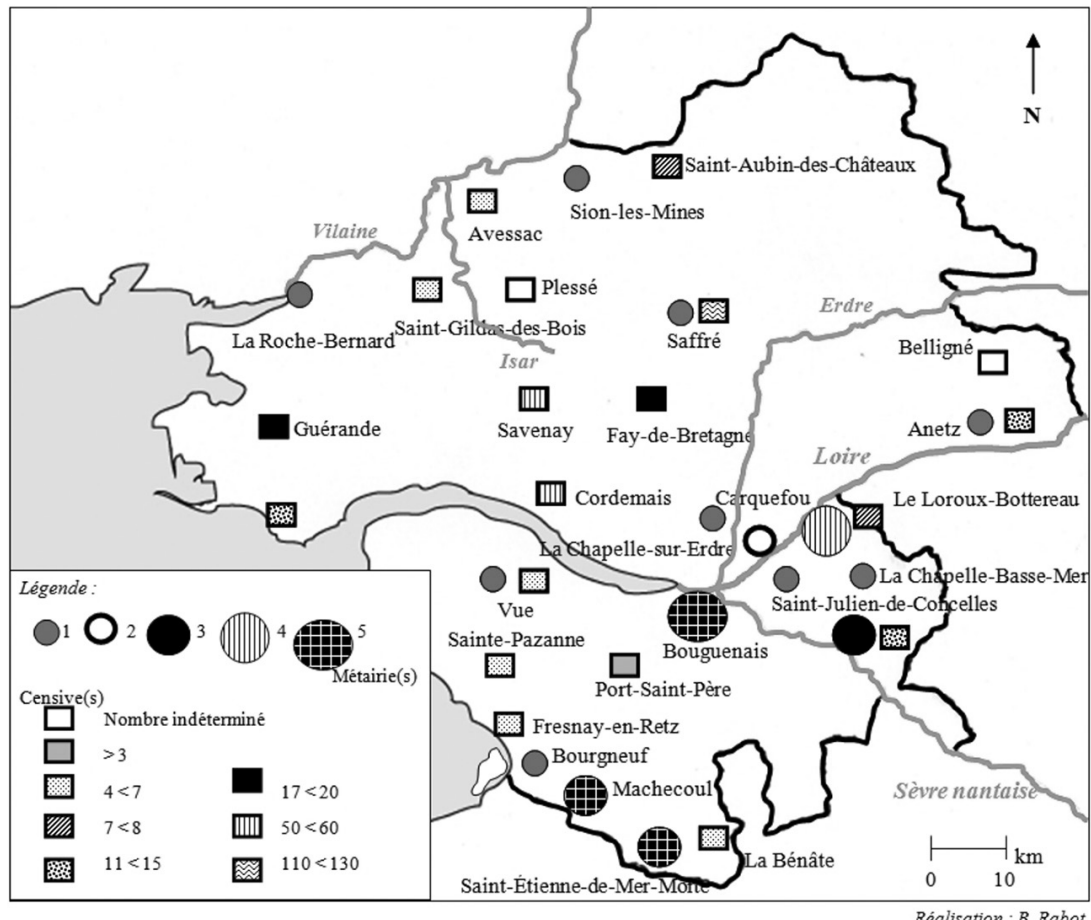

conservation des documents et l'apparition, dans les fonds disponibles, des premiers registres de comptes permettent de replacer les informations dégagées dans un contexte plus large, pour tenter de mieux comprendre les logiques d'implantation des structures agricoles.

Les censives, largement répandues à cette époque dans l'ensemble du comté, sont d'abord l'un des signes les plus visibles de l'importance de la petite propriété paysanne en Bretagne méridionale. Fondées sur le paiement du cens, qui leur a donné leur nom, les censives mettent en œuvre en réalité pour un même fonds une double propriété qui distingue l'usage de la possession du sol. Les tenanciers qui les exploitent détiennent la propriété utile; ils s'acquittent pour cela chaque année d'une taxe recognitive, le cens, réglé le plus souvent en numéraire. Le cens peut être accompagné, bien que le cas soit plus limité d'après les informations disponibles pour le XIV et la première moitié du $\mathrm{XV}^{\mathrm{e}}$ siècle, de redevances en nature avec des céréales et/ou des volailles ${ }^{42}$. La propriété éminente, symbolique, revient

42. Ibidem, p. 204-223. 
quant à elle au seigneur foncier qui continue de disposer d'une autorité sur la terre concédée. Le geste du paiement effectué par le tenancier sert à rappeler cette propriété et l'obéissance due à ce titre au seigneur foncier, comme ne manquent quasiment jamais de le rappeler les aveux et autres dénombrements.

Les censives sont attestées dans toutes les seigneuries, petites ou grandes. Elles sont des outils majeurs pour mettre en valeur les terres, les seigneurs n'hésitant pas à y recourir, quels que soient leur statut et l'étendue ou la diversité de leur patrimoine. Les censives sont toutefois beaucoup plus présentes dans les territoires dominés par les plus petits seigneurs ou sieurs que dans ceux placés sous l'autorité des châtelains ou des barons, à l'instar du pays de Retz, au sud de la Loire. Les sieurs, particulièrement nombreux en Bretagne méridionale, contrôlent une bonne part des ruraux du fait justement de leur dissémination sur l'ensemble du territoire ${ }^{43}$. Ils ne disposent pas, à la différence des seigneurs, de sources de revenus variées et lucratives en dehors de l'exploitation foncière, du fait de l'étroitesse de leurs prérogatives. Pour mettre en valeur leur patrimoine, relativement restreint, les sieurs recourent donc aux concessions de terres, autrement dit aux censives, ce qui explique pour partie leur maintien et leur dispersion en comté de Nantes.

D'autres facteurs doivent aussi être évoqués pour comprendre ce maintien des censives. Les terroirs de la moitié septentrionale du comté de Nantes sont essentiellement tournés vers la céréaliculture, qui demeure sans conteste l'activité agricole principale. Les aveux prennent toujours soin de présenter en premier les productions céréalières, pratiquées dans le cadre des tenures, avant d'énumérer les autres types de culture, telles les vignes, le plus souvent exploitées en complant ${ }^{44}$. Sorte de pariage entre un propriétaire foncier, qui concède à un preneur une parcelle plantée en ceps ou à planter dans un délai prescrit dans un bail, le complant procure des revenus beaucoup plus lucratifs pour les seigneurs/sieurs que ceux tirés des censives ${ }^{45}$. Les complants, quoique de meilleur rapport, ne remplacent toutefois pas les censives tant que les tenanciers s'acquittent correctement et régulièrement des redevances et autres droits pesant sur elles. Des signes d'évolution interviennent dans la seconde moitié du $\mathrm{Xv}^{\mathrm{e}}$ siècle, au moment où les tenanciers éprouvent justement de plus grandes difficultés à payer les rentes. Les complants connaissent alors une nette progression, surtout dans la partie sud du comté, au contact du Poitou.

43. BoISROUVRAY, Xavier du, " Nouvelle introduction à l'inventaire sommaire de la série E des archives départementales de Loire-Atlantique nécessaire à son utilisation ", dans MAîTRE, Léon, Inventaire sommaire des Archives départementales antérieures à 1790, t. 3.

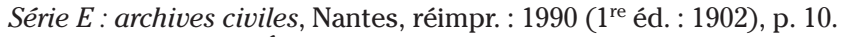

44. Guibert, Alain, Étude sur la vigne dans le comté nantais au Moyen Âge, mémoire de DES (dactyl.), Université de Nantes, 1970.

45. Ibidem, p. 29. Pour une remise en perspective du commerce du vin dans les échanges et des revenus que les seigneurs pouvaient en retirer : RENOUARD, Yves, "Le grand commerce du vin au Moyen Âge ", Revue historique de Bordeaux, 1952, p. 5-18. 
Les sources sont beaucoup plus discrètes pour tout ce qui a trait à l'élevage, qui constitue, comme nous l'avons dit plus haut, l'un des piliers des revenus des métairies. L'examen des comptes disponibles révèle l'importance des recettes tirées des bêtes dans les revenus seigneuriaux saisissables $^{46}$ (figure 2). La présence de forêts dans les environs de Saffré, de Machecoul, de Belligné et du Gâvre, parmi les plus vastes du comté, encourage cette activité avec les panages et les glandées organisés à grande échelle. Des métairies sont par ailleurs attestées dans ces mêmes zones. Les seigneuries plus modestes ne sont pas non plus en reste pour l'élevage, comme en attestent les registres de La Blanchardais (Vue) qui présentent, dans les années 1480-1490, des cheptels, certes plus réduits, mis en pâture dans des prés qui s'apparentent fort à des communaux ${ }^{47}$.

\section{Les zones de marches}

Les territoires aux confins de la Bretagne méridionale et des provinces françaises présentent d'autres traits que ceux que nous venons de décrire pour les espaces intérieurs. Au tournant des années 1450-1460, au moment où débute le vaste mouvement de reconstruction agraire entrepris en Anjou $^{48}$ et en Poitou ${ }^{49}$, les métairies des marches du comté connaissent des progrès marqués avec des mentions plus régulières dans les documents et la transmission de quelques baux. La conservation croissante d'aveux et de comptes permet de mieux cerner certains aspects de ce développement pour la seconde moitié du XV e siècle (figure $5^{50}$ ) même si, là encore, les informations demeurent disparates d'une seigneurie à l'autre.

46. Tous les revenus ne se trouvent en effet pas inscrits dans les registres de compte. Certains étaient directement perçus par les seigneurs, sans que les receveurs retranscrivent les sommes dans les cahiers (par exemple, Arch. dép. de Loire-Atlantique, 1 E 222 [1], fo $3 \mathrm{v}^{\circ}$ ), tandis que d'autres, comme les rentes en céréales, étaient inscrits dans des registres spécifiques, conservés à part et aujourd'hui perdus (les receveurs de La Blancharadais évoquent ainsi des comptes de " grèneterie ", disparus : par exemple, Arch. dép. de Loire-Atlantique, 1 E 223 [3], fo $11 \mathrm{r}^{\circ}$ ).

47. C'est ce qu'indique le compte rendu par le receveur de La Blanchardais (Vue) en 1491 : "Savoir pour les pasnages et affiages des bestes affiées es demaines de monseigneur en l'Isle de Veuz et ailleurs ou est de coustume d'affié [...] " (Arch. dép. de LoireAtlantique, Nantes, 1 E 223 [3], f $\mathrm{f}^{\circ} 8 \mathrm{v}^{\circ}$ ). Il atteste ainsi de la présence de 22 vaches et 6 juments mises en pâture en 1487 sur les prés du domaine de l'Île de Vue, 21 vaches et 6 juments pour l'année 1488, 19 vaches et 5 juments pour l'année 1489, 16 vaches et 3 juments pour l'année 1490 et 21 vaches et 4 juments pour l'année 1491 (Arch. dép. de Loire-Atlantique, Nantes, 1 E 223 [3], fo $8 \mathrm{v}^{\mathrm{o}}-10 \mathrm{v}^{\mathrm{o}}$ ). Ces chiffres doivent toutefois être maniés avec précaution : les paysans pouvaient en effet mettre en pâture leurs bêtes sur d'autres terres appartenant à d'autres seigneurs, qui n'étaient pas recensées dans les comptes de La Blanchardais (RАBOT, Brice, "La gestion d'une seigneurie rurale de 1429 à 1513. La comptabilité de la Blanchardais ", Histoire et Sociétés rurales, vol. n 45 , $1^{\mathrm{er}}$ semestre 2016 , p. 70 ).

48. LE MenÉ, Michel, Les campagnes angevines..., op. cit., p. 188-189.

49. MERLE, Louis, La métairie et l'évolution..., op. cit., p. 49-59, 68-70, 99-100.

50. Arch. dép. de Loire-Atlantique, B 125, B 126, B 127, B 129, B 130, B 131, B 842, B 843, B 1441, B 1446, B 1458, B 1482, B 1816, B 1817, B 1819, B 1821, B 1825, B 1829, B 1832 , 
Figure 5 - Répartition spatiale des structures agraires en comté de Nantes d'après les indications des sources issues des fonds des Archives départementales de Loire-Atlantique (années 1450-1510)

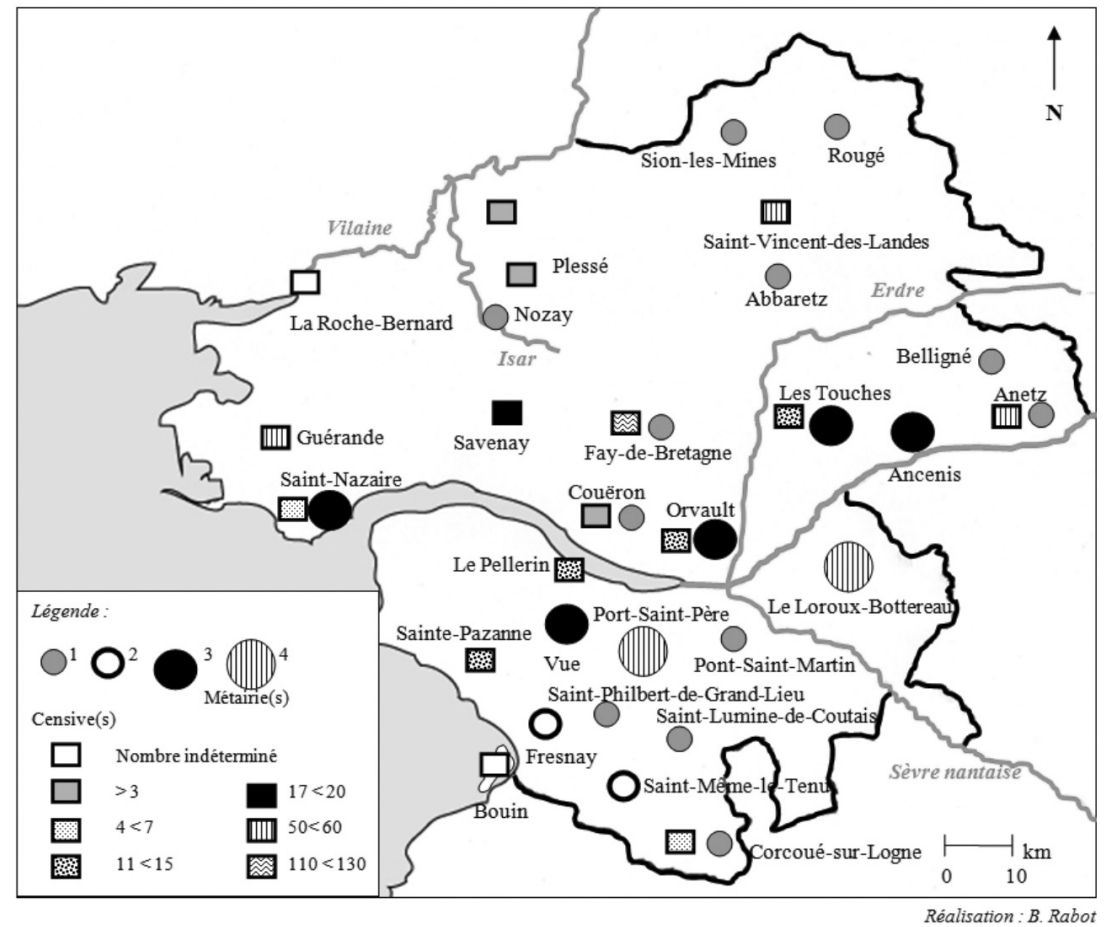

Les bordures frontalières concentrent nettement les métairies, sans que celles-ci supplantent, une fois encore, totalement les censives. Ce maintien des censives suggère les hésitations ou les difficultés rencontrées par les seigneurs/sieurs pour réorienter les structures agraires, en lien avec une modification/adaptation des structures du prélèvement ${ }^{51}$. La progression lente des métairies dans cette partie du comté ne doit pas non plus être limitée à la seconde moitié du $\mathrm{XV}^{\mathrm{e}}$ siècle. La comparaison avec la carte établie pour la période 1400-1440 (figure 3) permet de remettre

B 1836, B 1842, B 1843, B 1846, B 1848, B 1849, B 1851, B 1858, B 1859, B 1866, B 1870 , В 1874, В 1875, В 1881, В 1885, E 150, E 151, E 261, E 262, E 263, E 264, E 267, E 299, E 304, E 306, E 335, E 336, E 343, E 352, E 362, E 365, E 366, Е 387, E 451, E 458, E 470, E 471, E 472, E 473, E 483, Е 487, Е 489, Е 490, Е 495, Е 496, Е 508, E 514, E 517, E 524, E 557, E 596, E 600, E 606, E 607, E 608, E 610, 1 E 58, 1 E 64, 1 E 65, 1 E 155, 1 E 156, 1 E 165, 1 E 176, 1 E 179, 1 E 180, 1 E 181, 1 E 183, 1 E 217, 1 E 322, 1 E 333, 1 E 674, H 3, H 5, H 6, H 7, H 8, H 10, H 11, H 12, H 14, H 15, H 16, H 17, H 25, H 39, H 76, 21 J 13, 36 J 24, 217 J 22. Sont inscrits ici le nombre d'exploitations et de tenanciers/métayers cités dans les aveux et les dénombrements.

51. Structures du prélèvement seigneurial et structures agraires sont très étroitement liées (RABOT, Brice, Les structures seigneuriales..., op. cit., p. 189-250). 
les informations disponibles dans une perspective de temps plus long. Le rapprochement entre les cartes révèle que le mouvement de diffusion et de progression des métairies dans les zones bordières était déjà à l'œuvre dans la période précédente. Il reste en effet difficile de fixer une date plus précise pour le début de ces évolutions, faute de sources en nombre suffisant pour la fin du XIV ${ }^{\mathrm{e}}$ siècle, mais aussi faute d'indications relatives aux métairies dans les documents existants. Le développement des métairies s'affirme dans la partie du comté comprise entre l'Erdre et l'Anjou, là où sont implantées de larges zones de bois et des structures seigneuriales dotées de plus vastes prérogatives, telles la châtellenie de Belligné et la prévôté d'Ancenis ${ }^{52}$. L'autorité seigneuriale plus prégnante, bien que loin d'être insupportable pour les tenanciers si l'on en juge par les données transmises par les sources, fournit un premier élément d'explication au développement des métairies dans cette partie du comté.

Le changement d'échelle offre un autre regard. Au contact de l'Anjou à l'est, les seigneurs de cette portion du comté entretiennent des liaisons étroites, ne serait-ce que par les échanges commerciaux, avec leurs homologues angevins. Les seigneurs bretons sont ainsi influencés par le modèle angevin, même si les recompositions côté breton ne s'effectuent ni au même rythme, ni avec la même profondeur ${ }^{53}$. Le maintien des censives, qui coexistent toujours aux côtés des métairies à la veille du Xvi ${ }^{\mathrm{e}}$ siècle, souligne à quel point les structures agraires bretonnes restent marquées par une profonde inertie. Il en résulte une situation originale, où les métairies se développent lentement sans remplacer totalement les autres modes de faire-valoir. Les seigneurs bretons de la fin du Xve siècle ne disposent pas tous non plus des moyens nécessaires à la reconstruction des campagnes, ce qui explique, pour partie, la juxtaposition des modes de faire-valoir.

\section{Les métairies au sud de la Loire}

Le pays de Retz, qui domine la majeure partie de la rive sud de la Loire, offre un dernier sous-ensemble original dans le contexte du comté de Nantes, qui nous conduit à le présenter séparément. La seconde moitié du $\mathrm{XV}^{\mathrm{e}}$ siècle marque dans cette partie du comté un véritable tournant, indéniable, en impulsant un mouvement de substitution des métairies aux censives. Dominé par l'une des plus puissantes baronnies bretonnes, celle de Retz, le pays de Retz est aussi l'un des espaces les mieux documentés pour le comté de Nantes à la fin du Moyen Âge.

L'étude des fonds notariaux révèle l'importance des " accapareurs de terres " dans la constitution des métairies. Ces hommes agissent pour

52. Ces structures sont toutes deux placées sous la dépendance de la famille de Rieux, l'une des plus puissantes familles baronniales bretonnes à la fin du Moyen Âge (GuILLAUME, Gwenaël, Les Rieux : une famille de la haute noblesse bretonne aux XIV et XV siècles, thèse de doctorat (dactyl.), Université de Nantes, 2000, t. 2, p. 395).

53. RАвот, Brice, Les structures seigneuriales..., op. cit., p. 221-223, 565-566. 
leur seigneur ou, comme l'a montré Jean-Luc Sarrazin, pour leur propre compte $^{54}$. Jean Gauguet apparaît comme l'un des exemples les plus révélateurs à cet égard. Présenté comme procureur des moines de Buzay dans les actes qui nous sont parvenus, le frère Gauguet agit surtout " de façon très indépendante ", en son nom propre. Durant sa période d'activité, de 1438 à 1473, ce " procureur " nous a légué 99 actes, qui permettent de remettre en perspective les informations dégagées. Il procède par adjonctions successives en renforçant peu à peu la métairie de la Petite Angle, qu'il détient aux confins des paroisses de Cheix-en-Retz et de Rouans, en plein cœur du pays de Retz, par l'incorporation de terres exploitées auparavant en censives. L'assise foncière de la Petite Angle se trouve ainsi consolidée par des ajouts concentriques, opérés au départ au gré des circonstances, avant de devenir de plus en plus réfléchis et structurés dans la seconde moitié du $\mathrm{XV}^{\mathrm{e}}$ siècle. Jean Gauguet confie ensuite la métairie de la Petite Angle à de nouveaux exploitants, qui lui offrent des revenus plus intéressants que ceux des censives. Ces ressources lui permettent de poursuivre le remembrement opéré à son profit, tout en changeant en profondeur les structures agraires et les rapports entre propriétaire foncier et exploitants en développant le métayage. L'exemple de la Petite Angle n'est pas isolé. D'autres sont également attestés, toujours dans les environs de Cheix-enRetz, de Rouans, mais aussi au nord-ouest, au Pellerin, ou à l'intérieur des terres, à Saint-Philbert-de-Grand-Lieu ${ }^{55}$.

Jean Gauguet n'est pas seulement procureur des moines de Buzay, il est aussi seigneur foncier. Détenteur du domaine du Périer, à une quinzaine de kilomètres à l'ouest de Buzay, à Sainte-Opportune-en-Retz, il bénéficie de disponibilités suffisantes, avec celles de l'abbaye de Buzay, pour mener à bien ces opérations. Véritable " entrepreneur " foncier, il tire profit de la demande monétaire des paysans, sans cesse croissante $\mathrm{au} \mathrm{XV}^{\mathrm{e}}$ siècle, pour dissimuler des prêts sous la forme d'achats de rentes et d'achats de bienfonds, qu'il récupère lorsque les preneurs sont dans l'incapacité d'honorer leurs engagements. Les stratégies mises en œuvre par Jean Gauguet s'appuient donc sur les événements et le poids de la conjoncture, de plus en plus heurtée à partir des années 1450-1460, qui accélèrent un processus enclenché au moment où les campagnes de Bretagne méridionale sortaient du marasme de la fin du XIV ${ }^{\mathrm{e}}$ siècle.

Les seigneurs et leurs intermédiaires tirent profit des difficultés grandissantes de la fin du $\mathrm{XV}^{\mathrm{e}}$ siècle pour reprendre, en jouant sur les clauses de réméré ${ }^{56}$, des tenures aux exploitants incapables de s'acquitter de leurs

54. SARRAZIN, Jean-Luc, " Les activités... ", art. cité, p. 136.

55. Arch. dép. de Loire-Atlantique, E 1071, E 1073, E 1074.

56. Désigne la " clause d'un contrat de vente par laquelle le vendeur se réserve le droit de racheter son bien dans un délai convenu en remboursant à l'acquéreur le prix principal et les frais de son acquisition. [...]": Outils et Ressources pour un Traitement Optimisé de la Langue (Ortolang), Centre National de Ressources Textuelles et Lexicales. Disponible sur : [http://www.cnrtl.fr/definition/r\%C3\%A9m\%C3\%A9r\%C3\%A9] (consulté le 4 octobre 2016). 
dettes ou de racheter leurs terres. Les métairies prennent peu à peu le relais des censives en regroupant, de manière lente et progressive, des parcelles jusque-là dispersées ou en établissant, autour de noyaux préexistants, des exploitations plus grandes. Ce processus, qui s'étale sur plusieurs décennies, a été parfaitement décrit et analysé par Jean-Luc Sarrazin ${ }^{57}$, dans ce qu'il appelle le processus de "rassemblement des terres par des accapareurs". En se penchant sur l'étude plus précise de la région de Saint-Philbert-deGrand-Lieu, ainsi que sur celle, plus au nord, du Pellerin, Jean-Luc Sarrazin a dégagé quelques grandes logiques qui, il faut bien le dire, restent difficilement transposables à d'autres zones, faute de disposer des fonds notariaux nécessaires pour pouvoir entreprendre le même type d'étude. Ce travail est donc d'autant plus précieux et riche en enseignements.

La présence de châtellenies à Pornic, à Machecoul, à La Bénâte ou encore aux Huguetières (La Chevrolière) renforce le poids de l'autorité seigneuriale, sans que celle-ci soit insupportable pour les paysans. Les châtelains encadrent plus strictement les hommes ${ }^{58}$, même si cet encellulement reste difficile à mettre en œuvre au quotidien dans le cadre d'habitats dispersés en hameaux et en petits villages ${ }^{59}$. Les métairies, que les châtelains contribuent à développer et à étendre, permettent justement de mieux fixer les hommes autour d'exploitations agricoles sur lesquelles le contrôle seigneurial s'exerce plus facilement.

Le pays de Retz est enfin traversé par de nombreux flux, qui accentuent et accélèrent les confrontations d'influences en provenance du reste de la France ${ }^{60}$. Les sires de Retz et leurs vassaux entretiennent des liaisons étroites avec les seigneurs poitevins au travers des versements de rentes. Les comptes de La Blanchardais, seigneurie foncière vassale de Retz, indiquent ainsi le paiement de redevances en numéraire et en nature aux receveurs des seigneurs de La Guerche ${ }^{61}$. Les châtelains des Huguetières, également vassaux de la baronnie de Retz, sont aussi amenés à verser des rentes à des receveurs/seigneurs poitevins pour les fiefs détenus dans les marches ${ }^{62}$, entretenant ainsi des liaisons d'homme à homme qui facilitent la confrontation des expériences.

Au total, l'absence ou la présence de grandes châtellenies ou de baronnies, la proximité de forêts, la part variable de l'élevage dans les activités

57. SARRAZIN, Jean-Luc, "Les activités... ", art. cité, p. 146-148.

58. Ibidem, p. 149-153.

59. PIснот, Daniel, Le village éclaté : habitat et société dans les campagnes de l'Ouest au Moyen Âge, Rennes, PUR, 2002, p. 188-203.

60. Les métairies bretonnes présentent ainsi de nombreuses similitudes avec celles du

Poitou (RABот, Brice, Les structures seigneuriales..., op. cit., p. 221).

61. Par exemple : " [...] Premier, compte cedit receveur avoir poié à Symon Bernart, receveur de La Guierche, tesmoign quictance dudit Bernart, signée à sa requeste de la main de Pierres Corbeau, donnée le $\mathrm{XX}^{\mathrm{e}}$ jour de janvier l'an mil $\mathrm{IIII}^{\mathrm{CC}}{ }_{\text {IIII }}^{\mathrm{XX}} \mathrm{XV}$ VII boexeaux froment [...]" (Arch. dép. de Loire-Atlantique, 1 E 223 (4), fo $21 \mathrm{r}^{\circ}$ ). D’autres rentes en numéraire sont attestées dans le même registre : Idem, fo $19 \mathrm{r}^{\mathrm{o}}, 25 \mathrm{v}^{\mathrm{o}}$.

62. SADDIER, Évelyne, Une seigneurie rurale..., op. cit., p. 11, 14, 103-105. 
agricoles, les influences extérieures sont autant d'éléments à considérer pour mieux comprendre l'essor des métairies à la fin du Moyen Âge en comté de Nantes.

\section{Des évolutions à remettre en perspective}

Les dernières remarques montrent à quel point l'étude des structures agraires permet d'approcher, de manière plus large, les bouleversements de la fin du Moyen Âge. Il faut donc remettre en perspective les évolutions présentées en mêlant les approches et les regards. Une série de questions au croisement de l'histoire et de la géographie éclaire alors utilement le propos : pour quelles raisons les réorientations agraires ne suivent-elles pas le même rythme selon les territoires étudiés? Quelles sont les conséquences de ces recompositions sur la mise en valeur agricole et l'organisation spatiale, notamment en matière de peuplement?

\section{Les crises de la fin du Moyen Âge}

Les stratégies mises en œuvre par le frère Gauguet ont permis de dégager un premier levier, essentiel, utilisé par les seigneurs à la fin du Moyen Âge avec la crise économique et monétaire. Réorienter les structures agraires est, dans ces conditions, d'autant plus nécessaire que les seigneurs eux-mêmes doivent faire face aux besoins et aux tensions, en même temps que leurs exploitants. La fin du Moyen Âge est en effet marquée par la " crise des revenus seigneuriaux". Cette épreuve est sans conteste l'une des plus graves secousses à laquelle les seigneurs ont eu à faire face ${ }^{63}$. La Bretagne n'échappe bien sûr pas à cette crise, mais les répercussions et la chronologie ne sont toutefois pas tout à fait identiques à celles observées dans les provinces voisines. L'essentiel de la crise ne se produit pas au $\mathrm{XIV}^{\mathrm{e}}$ siècle, comme dans les provinces françaises de l'Ouest avec la progression des Anglais et les ravages causés par les routiers en pleine guerre de Cent $\mathrm{Ans}^{64}$, mais bien dans la seconde moitié du XV ${ }^{\mathrm{e}}$ siècle, à partir des années 1460 surtout, au moment où la politique ducale bretonne entre en opposition frontale avec celle du roi de France ${ }^{65}$. Deux épisodes mettent particulièrement à mal le fragile équilibre de l'économie bretonne : l'incursion française opérée en 1468 à partir de la région d'Ancenis, dont les effets se font ressentir loin à l'intérieur du comté ${ }^{66}$ et, plus encore, le conflit de

63. "C'est par une crise des revenus seigneuriaux que se termine le Moyen Âge et s'ouvrent les Temps modernes ", cité dans BLOCH, Marc, Les caractères originaux de l'histoire rurale française, Paris, A. Colin, rééd. : 1988 (1 ${ }^{\text {re }}$ éd. : 1931), p. 143.

64. Minois, Georges, Nouvelle histoire de la Bretagne, Paris, Fayard, 1992, p. 299-308; Leguay, Jean-Pierre et Martin, Hervé, Fastes et malheurs..., op. cit., p. 98-108.

65. RАвот, Brice, Les structures seigneuriales..., op. cit., p. 445-481.

66. Ibidem, p. 454-455. Voir aussi LE MENÉ, Michel, « La conjoncture économique angevine sous le règne de Louis XI ", dans CHEVALIER, Bernard, ConTAmine, Philippe (dir.), La France de la fin du $\mathrm{XV}^{e}$ siècle : renouveau et apogée. Économie - pouvoir-arts. Culture et conscience nationales, Paris, CNRS, 1985, p. 51-60. 
1487-1491, qui aboutit au rattachement définitif de la Bretagne à la couronne de France, insérant le duché dans de nouveaux circuits d'échanges ${ }^{67}$. La Bretagne méridionale, le comté de Nantes en particulier, devient dès lors une cible et un objectif stratégique pour les belligérants, ce qui accentue un peu plus le poids des désordres et des destructions.

La crise politique et militaire ébranle les structures agraires, mais elle n'est pas la seule. Les besoins liés aux guerres, de plus en plus pressants aux XIV et $\mathrm{XV}^{\mathrm{e}}$ siècles surtout, conduisent les souverains à manipuler la valeur de la monnaie pour tenter de financer des dépenses toujours croissantes ${ }^{68}$. Les mutations monétaires qui en résultent ont des effets d'autant plus pernicieux qu'elles désorganisent en profondeur les structures économiques. La valeur des rentes en numéraire s'effrite, tandis que le poids de la fiscalité ducale bretonne s'accroît à mesure que les tensions avec la France se font plus pressantes sous le gouvernement de François II (1458$1488)^{69}$. Véritables gangrènes pour les seigneurs ruraux, les contrecoups des mutations monétaires sont accentués par les structures du prélèvement. Fondés pour une grande part, quoique inégale selon l'étendue et la diversité des prérogatives et droits seigneuriaux, sur les censives, les revenus fonciers ne cessent de s'éroder sans que les seigneurs puissent réévaluer les montants dus par les tenanciers du fait de la nature du cens, fixe et immuable tant que le tenancier preneur ou ses héritiers occupe(nt) la terre concédée, la cultive(nt) et s'acquitte(nt) des droits dus à ce titre. Nombre de seigneurs se trouvent placés dans une impasse d'autant plus grave que la conjoncture nécessite de réorienter rapidement les structures du prélèvement pour tenter justement de faire face. Certains choisissent alors de se tourner vers d'autres systèmes d'exploitation que les censives, en accordant une plus grande part aux redevances en nature. Les métairies répondent parfaitement à cette exigence et progressent particulièrement dans les territoires où d'autres redevances partiaires étaient fréquemment exigées $^{70}$ (figure $6^{71}$ ).

67. TOUCHARD, Henri, Le commerce maritime..., op. cit., p. 306.

68. Leguay, Jean-Pierre et MarTin, Hervé, Fastes et malheurs..., op. cit., p. 394-395; Rabot, Brice, Les structures seigneuriales..., op. cit., p. 445-481; CoATIVY, Yves, La monnaie bretonne..., op. cit., p. 370-372, 392-393.

69. MinoIs, Georges, Nouvelle histoire..., op. cit., p. 334-337; CoRnETTE, Joël, Histoire de la Bretagne et des Bretons, t. 1 : Des âges obscurs au règne de Louis XIV, Paris, Seuil, 2005, p. 327328, 384-391; CinTRÉ, René, Les marches de Bretagne au Moyen Âge..., op. cit., p. 145-161.

70. RАвот, Brice, Les structures seigneuriales..., op. cit., p. 209-218; Idem, " Pour une nouvelle approche du prélèvement seigneurial. L'exemple du pays de Retz à la fin du Moyen Âge ", Annales de Bretagne et des Pays de l'Ouest, 2016, t. 123, nº 1, p. 96-100. Sur les champarts et les terrages, voir aussi GALLET, Jean, Seigneurs et paysans bretons..., op. cit., p. 246-247.

71. Arch. dép. de Loire-Atlantique, B 842, B 843, B 1441, B 1816, B 1817, B 1825, B 1836, B 1843, B 1854, B 1866, B 1870, B 1875, E 299, E 304, E 329, E 490, E 522, E 607, 1 E 65, 1 E 183, 1 E 217, H 10, H 17, H 76; Cartulaire des sires de Rays (1160-1449), BLANCHARD, René (éd.), Archives historiques du Poitou, t. 30, Poitiers, 1899, pièce cccxII, p. 448. 
Figure 6 - Répartition spatiale des indications de champart et terrage aux $X I V^{e}$ et $X V^{e}$ siècles relevées dans les fonds des Archives départemen-

\section{tales de Loire-Atlantique}

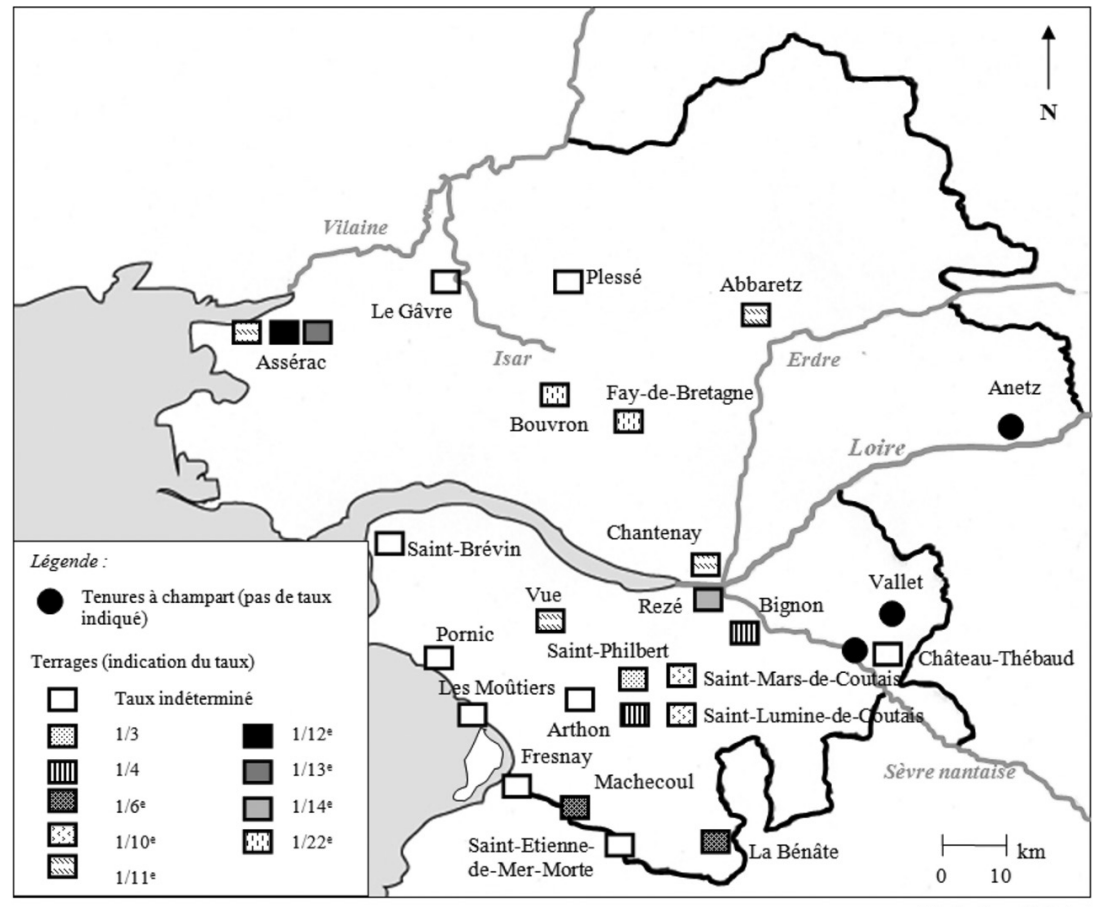

Réalisation : B. Rabo

Le pays de Retz ou les marches orientales avec l'Anjou concentrent ainsi métairies et tenures à champart/terrage, qui se complètent utilement tout en diversifiant les redevances versées par les tenanciers. Les rentes perçues en nature ont le très net avantage de ne pas être dépendantes des variations de la monnaie. Elles sont, en revanche, liées aux marchés, puisque les seigneurs doivent, une fois qu'elles ont été collectées, les écouler à l'extérieur. Pour contourner cette difficulté, certaines peuvent être stockées avant d'être remises dans les circuits d'échanges, en fonction des besoins ${ }^{72}$, pour éviter tout effondrement des cours, ce qui ruinerait les efforts entrepris.

\section{Les influences extérieures}

La succession de crises de différentes natures, dans la seconde moitié du $\mathrm{XV}^{\mathrm{e}}$ siècle, est un autre trait original du comté de Nantes dans l'Ouest français à la fin du Moyen Âge. Elles coupent alors le comté du Poitou et

72. RАВот, Brice, « Pour une nouvelle approche... », art. cité, p. 110-111. 
de l'Anjou qui, alors que les campagnes bretonnes sortent tout juste du marasme, entreprennent un vigoureux mouvement de reconstruction qui culmine à la veille du XvI ${ }^{\mathrm{e}}$ siècle ${ }^{73}$. Le décalage chronologique de l'évolution de la zone bretonne ne marque pas pour autant une rupture brutale. Malgré les difficultés persistantes, les seigneurs/sieurs prennent en effet des décisions qui orientent les structures agraires dans des directions proches de celles suivies par leurs homologues angevins ou poitevins. Se pose alors la question des influences extérieures et de leur part dans les réorientations agraires bretonnes à la fin du Moyen Âge.

La documentation disponible ne permet qu'indirectement de saisir les traces des influences extérieures. Les baux, qui organisent et définissent les responsabilités et les obligations du seigneur à l'égard du métayer et du métayer à l'égard du seigneur s'avèrent précieux. Malheureusement, ils sont très rares pour le comté de Nantes et n'apparaissent véritablement qu'à la fin du $\mathrm{Xv}^{\mathrm{e}}$ siècle ${ }^{74}$. Leur étude permet néanmoins de remettre en perspective les informations concernant les métairies bretonnes en les confrontant avec celles des espaces voisins. La plupart des baux fixent une durée de concession pouvant aller jusqu'à neuf ans ${ }^{75}$, à l'instar de ce que Michel Le Mené observe pour l'Anjou au Xve siècle ${ }^{76}$. Aucune indication au sujet du taux du prélèvement n'apparaît toutefois pour la fin du Xve siècle. Il faut donc se tourner vers les baux postérieurs, conservés en nombre croissant pour le $\mathrm{XVI}^{\mathrm{e}}$ siècle, pour saisir de tels ordres de grandeur, généralement fixés au quart des récoltes ou des ventes des produits issus des métairies ${ }^{77}$.

Comme en Anjou ou en Poitou, les métairies qui se développent à la fin du Xve siècle contribuent à renforcer les revenus seigneuriaux. La part des recettes tirées des métairies dans les ressources totales observables dans les comptes est importante jusqu'au début des années 1480, avant de

73. MERLE, Louis, La métairie et l'évolution..., op. cit., p. 72-73.

74. RАВОт, Brice, Les structures seigneuriales..., op. cit., p. 123-124.

75. C'est ce qu'indiquent, par exemple, les comptes de la châtellenie des Huguetières : " [...] Item, compte ledit chastellain de la métaerie de La Guillaudiere, affermée à Jehan Thebaud et Guillaume Gouillandeau pour neuff ans et neuff levées parfaictes et acomplies, comançsans à la feste de la Pentecouste l'an mil IIII ${ }^{\mathrm{C}}$ IIII ${ }^{\mathrm{XX}}$ et unze et y finissans lesdiz neuff ans révoluz à la somme de seix escus d'or par chacun an, oultre 25 soulz de rante deuz chacun an sur ladicte métaerie et par chacun desdits neuff ans, ouict biains, chacun garny de quatre beuffs et une charrette, et pour les gouverner et pour les négoces de ladicte chastellenie et rendront ladite métaerie ensemancée à la fin de ladite ferme et garnie d'angreix et partie des terres guerrettées [...]" (Arch. dép. de Loire-Atlantique, Nantes, E 503 [1], f ${ }^{\circ}$ 19).

76. LE MENÉ, Michel, Les campagnes angevines..., op. cit., p. 189.

77. LE MEnÉ, Michel, "Les redevances à part de fruits... ", art. cit, p. 19-20; GALLET, Jean, Seigneurs et paysans bretons..., op. cit., p. 236; PІснот Daniel, "Le prélèvement seigneurial dans l'ouest de la France (XI ${ }^{\mathrm{e}}$-XIII ${ }^{\mathrm{e}}$ siècle) ", dans Bourin-DERruAu, Monique et MARTínEZ SoPEnA, Pascual (dir.), Pour une anthropologie du prélèvement seigneurial dans les campagnes médiévales (XI'-XIV siècles). Réalités et représentations paysannes, colloque tenu à Medina del Campo du 31 mai au 3 juin 2000, Paris, Publications de la Sorbonne, 2004, p. 620; RABOT, Brice, Les structures seigneuriales..., op. cit., p. 222-223. 
Figure 7 - Part des revenus tirés des métairies dans les recettes totales inscrites dans les comptes de la prévôté d'Ancenis (1463-1501)

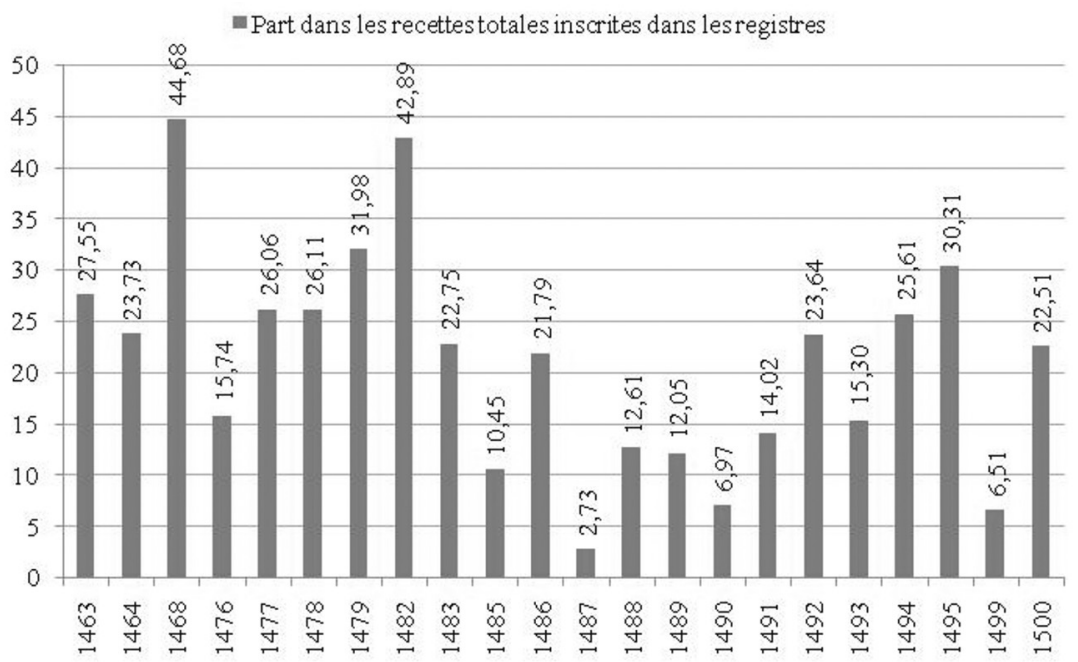

connaître un très net fléchissement lors de la crise de 1487-1491 (figure $7^{78}$ ). Les contrecoups des crises sont lourds de conséquences. La part des métairies dans les recettes totales ne retrouve pas, à la veille du XVI ${ }^{\mathrm{e}}$ siècle, les niveaux antérieurs aux crises des années 1470-1480, preuve à la fois de l'intensité de la crise, qui met à mal en profondeur les structures du prélèvement, mais preuve aussi de la difficulté rencontrée par les seigneurs à la fin du $\mathrm{XV}^{\mathrm{e}}$ siècle pour trouver de nouveaux preneurs. Les métairies, comme les censives, doivent alors faire face à des ajustements structurels, qui entraînent un repli des revenus au début du $\mathrm{XvI}^{\mathrm{e}}$ siècle.

Les traits observés pour la prévôté d'Ancenis se retrouvent pour la châtellenie de Belligné (fig. $8^{79}$ ). Les lacunes chronologiques empêchent toutefois de saisir avec précision les rythmes et les modalités d'évolution. Les revenus tirés de la métairie sont beaucoup plus aléatoires à Belligné que dans la région d'Ancenis, du fait de l'importance de l'élevage. Les revenus de l'année 1494 sont ainsi exclusivement constitués par les redevances tirées de l'élevage ${ }^{80}$. Comme dans la prévôté d'Ancenis, les revenus de la métairie de Belligné s'érodent à la fin des années 1490 et au début des années 1500, en lien avec la réorientation des flux et des circuits commerciaux. Les revenus ne s'effondrent toutefois pas complètement : le niveau

78. Arch. dép. de Loire-Atlantique, E 261 (3 à 5), E 262 (1 à 4), E 263 (1 à 4), E 264 (1 à 3).

79. Arch. dép. de Loire-Atlantique, E 261 (3 à 5), E 262 (1 à 4), E 263 (1 à 4), E 264 (1 à 3).

80. Les revenus totaux tirés de la métairie en 1494 s'élèvent à 27 livres 11 sous 8 deniers, dont 21 livres tirées de l'élevage, soit 76,13\% des " recettes incertaines " totales (Arch. dép. de Loire-Atlantique, E 268 (3). 
Figure 8 - Part des revenus tirés de la métairie dans les " recettes incertaines inscrites dans les comptes de la châtellenie de Belligné (1454-1504)

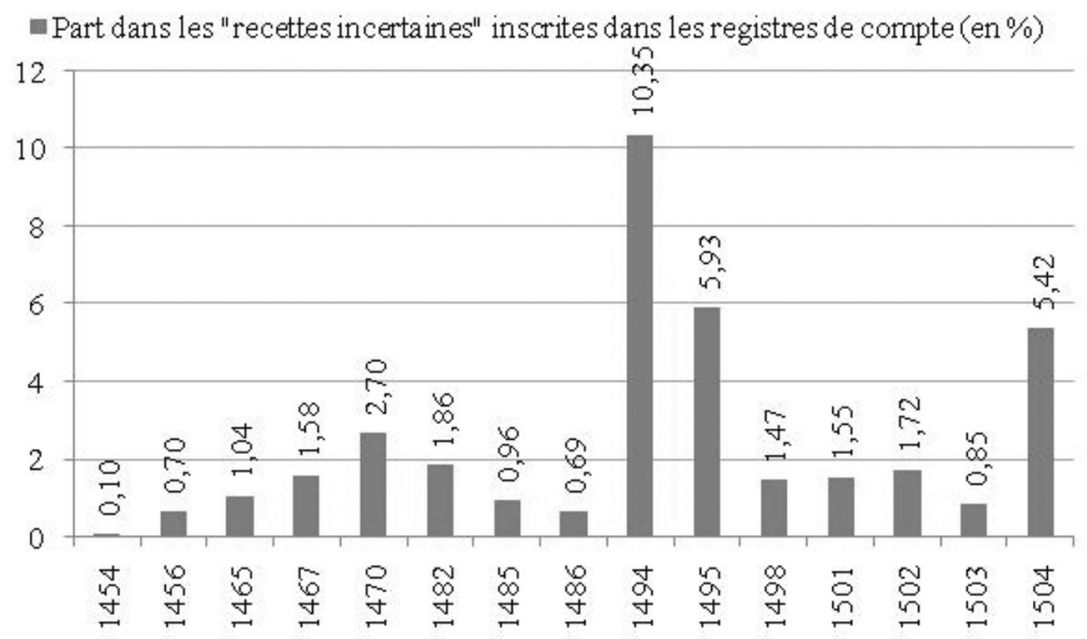

des années 1498, 1501, 1502 et, dans une moindre mesure, 1503, correspond en effet à celui observé dans les années 1460-1480. Les structures du prélèvement pour la métairie de Belligné ne semblent pas, dans ces conditions, avoir connu de profonds bouleversements après les crises de la fin du Xve siècle. Les métayers savent aussi trouver les moyens nécessaires pour rétablir la situation rapidement, permettant de stabiliser la part des revenus tirés de leur exploitation dans les " recettes incertaines " totales de la seigneurie.

Les modalités ou les enjeux que nous venons de décrire présentent de fortes similitudes avec ceux observés dans les provinces françaises. Le comté de Nantes ne reflète-t-il pas, plus largement, le partage entre une France du nord et une France du sud où le métayage se développe plus fermement que dans la moitié nord de la France? La réponse à cette question renvoie aux débats des années 1960-1980 sur la question des redevances à parts de fruits et de leur importance dans les revenus seigneuriaux, plus précisément dans l'ouest de la France ${ }^{81}$. Les éléments précédents soulignent l'originalité du comté de Nantes tant du point de vue breton, avec le contexte de marches, que du point de vue de l'Ouest français, avec une chronologie des crises décalée. Dans ces conditions, le comté est un espace singulier de structures agraires à la jonction d'impératifs opposés - reconstruire les campagnes dans un contexte d'instabilité monétaire et de réorientation des circuits économiques et commerciaux -, voire contra-

81. LE MENÉ, Michel, "Les redevances à part de fruits... ", art. cité. 
dictoires - maintien d'une petite propriété paysanne affaiblie par les crises du seconde $\mathrm{XV}^{\mathrm{e}}$ siècle, mais étroitement liée aux sieurs qui continuent de dominer ces territoires.

Qu'en est-il de la taille des métairies? Sont-elles, comme en Anjou ou en Poitou, de vastes surfaces, plus étendues que les censives? Nous nous heurtons là, une nouvelle fois, au silence trop fréquent des sources. Les informations disponibles sont très succinctes et, lorsqu'elles existent, survient le problème redoutable de la conversion des mesures anciennes en système métrique. Ce souci est d'autant plus grand que nous ne disposons que de renseignements épars et limités, qui plus est pour l'époque moderne ${ }^{82}$, alors que nous savons par ailleurs que les mesures anciennes étaient régulièrement revues et corrigées ${ }^{83}$. Les comptes n'apportent pas non plus d'informations sur les superficies. Tout juste peut-on saisir, au détour d'aveux, l'aire de quelques parcelles, de l'ordre de quelques arpents, réunies ensuite aux exploitations déjà constituées ${ }^{84}$. Les mouvements de remembrement, lents et complexes, accentuent un peu plus cette difficulté à saisir les surfaces des métairies avec l'espacement des aveux et l'absence de données pour toutes les terres. Il n'est donc pas possible d'apporter d'estimation fiable en dehors des seules études de cas, ce qui explique aussi pour partie l'absence de travaux historiques consacrés à ce sujet pour cette zone et cette période.

\section{Métairies et répartition des hommes}

Les métairies ne doivent pas être réduites à des systèmes d'exploitation agricoles; elles contribuent aussi, de manière plus large, à organiser les territoires sur lesquels elles s'implantent en fixant les hommes et en exerçant sur eux un contrôle plus étroit, ne serait-ce que par la présence du manoir dont elles dépendent, situé à leur proximité immédiate comme le laissent entendre les sources ${ }^{85}$. Elles sont, en ce sens, un instrument supplémentaire aux mains des seigneurs pour encadrer les paysans dans le contexte particulier du bocage breton ${ }^{86}$. Précisons toutefois que cet

82. Sur ce sujet précisément, voir Ibidem, p. 131-142, en particulier le tableau n ${ }^{\circ} 5$, p. 140-141. Pour établir des conversions les historiens disposent d'un seul ouvrage pour le comté de Nantes : DEMolon, Manuel décimal de l'arpenteur, Nantes, 1802 (recueil établi au XVIII ${ }^{\mathrm{e}}$ siècle).

83. GALLET, Jean, « Une mesure ancienne dans la paroisse de Baden : la mesure caignarde (XV"-XVIII ${ }^{\mathrm{e}}$ siècles) ", Bulletin de la société polymathique du Morbihan, 1972, p. 53.

84. Pour reprendre l'exemple du frère Gauguet, sur 69 parcelles achetées (pour lesquelles nous disposons d'informations suffisantes), 47 sont d'une superficie égale ou inférieure à 2 boisselées de terre, autrement dit 30 ares ou moins. Voir à ce sujet SARRAZIN, Jean-Luc, "Les activités... ", art. cité, p. 146.

85. Par exemple : «[...] Item le herbregement et mettaerie de la Pulliere o toutes ses appartenances tant en terres arables, non arables, prez, boais, pastureaux, marais, landes, que toutes autres chouses [...] ", extrait de l'aveu rendu le 15 avril 1390 par Sevestre de Rezé (Arch. dép. de Loire-Atlantique, E 352).

86. Pichot, Daniel, Le village éclaté..., op. cit., p. 224-226; RENARD, Jean, "Les paysages agraires du sud-est du Massif armoricain ", Enquêtes rurales, n 2, 1997, p. 113. 
encellulement est loin d'être très contraignant. Les aveux ou les comptes montrent indirectement que les métayers, comme les tenanciers du reste, disposent d'une certaine latitude dès lors qu'ils s'acquittent régulièrement et correctement des rentes dues. Derrière ces grands traits généraux, deux grandes orientations peuvent être plus précisément distinguées dans l'organisation des territoires, de part et d'autre de la Loire.

Au sud de la Loire, les métairies, désignées comme telles dans les sources ou sous le nom de "gagneries ", sont organisées autour du siège d'exploitation, avec des bâtiments séparés. La perte aujourd'hui des repères spatiaux, parfaitement connus des contemporains, d'où l'absence de références plus précises dans les sources, empêche d'aller plus loin dans les localisations. De même, il n'est fréquemment pas possible d'établir un lien direct entre taille des exploitations et taille des métairies pour la fin du Moyen Âge, faute d'indications et d'ordres de grandeur pour les équivalences :

" Item, les herbrégemens, gaigneries et vilages, nommés la métaierie du Chaffaut, La Melerie, La Conseillerie et La Rengeonnerie, tenues lesdictes gaigneries les unes les autres o touz leurs fons, boais, prez, rivages, terres, sauzaies et $o$ toutes leurs aultres appartenances quelxcomques. Et ce que je ay et prans en métérie, tant en vignes, prez, terres, sauzaies, deniers de rente que en toutes aultres chouses quelxcomques. Item, mon molin, nomé le molin de La Choammere, près celle métérie $[\ldots]^{87}$."

Nous ne pouvons pas non plus connaître le nombre exact de personnes vivant sur le fonds de la métairie. Seul le preneur, le métayer, est cité dans les sources avec, beaucoup plus rarement, mention de ses proches ou consorts sans autre précision ${ }^{88}$.

D'autres éléments peuvent en revanche être plus fermement saisis et établis. À Saint-Père-de-Bouguenais, dans la seigneurie du Chaffaut par exemple, la métairie se situe à proximité immédiate du moulin banal, en pleine réserve seigneuriale ${ }^{89}$. Le seigneur, Bertran du Chaffaut, ne manque pas dès lors d'y affirmer son autorité, qu'il prend soin de coucher par écrit, tout en exerçant un contrôle d'autant plus étroit sur la métairie et la fréquentation du moulin qu'il vit à proximité. Les métairies sont dans ces conditions de véritables piliers de l'autorité seigneuriale, en prise directe avec les lieux de sociabilité villageoise que sont les édifices banaux ${ }^{90}$.

87. Extrait de l'aveu rendu le 12 décembre 1451 par Bertrand du Chaffaut (Arch. dép. de Loire-Atlantique, E 150).

88. Par exemple : "Item, seze hommées de prez appartenant à mes mestaiers de La Guisaudiere, de La Marteliere, de La Giraudiere et de celle que tient ledit Lemariot. Item, la meson où demeure mon mestaier de La Giraudiere et ung [...] qui contient une bouexellée ou environ. Item, trente septerées de terre laborable que labore mon mestaier de Lerriere et de La Barboniere. [...]", extrait de l'aveu rendu le 26 avril 1447 par Lepart de La Jumelière (Arch. dép. de Loire-Atlantique, B 1842).

89. C'est ce qu'indique l'aveu rendu le 12 décembre 1451, qui précise : " la métairie de mondit houstel [...]" (Arch. dép. de Loire-Atlantique, E 150).

90. L'historiographie récente insiste davantage sur le rôle d'outils à la disposition des communautés rurales que sur le côté coercitif des édifices banaux (ARNoux, Mathieu, Le 
Au nord de la Loire, les quelques exemples saisissables pour la fin du Moyen Âge présentent d'autres caractères. Appelées là encore " gaigneries ", les métairies sont, dans cette partie du comté, regroupées en deux ou trois exploitations :

«Item, y a trois gaigneries de terres laborables, contenentes ensemble quarente journaux ou environ, esquelles gaigneries croyst communs ans oultre le droit du mestayer en une desdictes gaigneries quant elle est en labour par froment, dix bouexeaulx, par avaine grosse, vingt et quatre bouexeaulx, par avaine menue, neuf bouexeaulx ou environ, le tout mesure d'Ancenis $[\ldots]^{91}$."

ou encore :

"Premier, en la parroesse de Saint-Nezaire, mon herbrégement nommé herbrégement de Cleuz o touz ses clostures, relaex, courtilz, boaies, garenes, gaigneries, sises entre le herbrégement, courtilz et boays de monsire Pierres de Misnillac, à cause de ma dame Jahane de Cleuz, sa compaigne, d'une part, et la meson Guillo [...] d'autre et entre la Ville Hullin et la meson Estienne Paqueau $[\ldots]^{92}$."

Une telle organisation laisse penser à des exploitations plus grandes, plus dispersées aussi, qui regrouperaient plus de personnes en leur sein. Aucun élément n'apparaît à ce sujet dans les sources. Situées parfois à l'écart des autres terres, à l'instar de la métairie détenue par Jahen de Cleuz à Saint-Nazaire, les gagneries se trouvent placées au cœur d'un bocage à larges mailles, avec des superficies de l'ordre de plusieurs dizaines de journaux, à proximité de courtils et de bois, autant d'atouts pour l'élevage ou la vente du bois à l'extérieur. Les métairies, ou gagneries, s'insèrent donc davantage que les censives dans une économie tournée vers les échanges avec l'extérieur. Ceci leur permet d'assurer leur développement et leur extension à la fin du $\mathrm{Xv}^{\mathrm{e}}$ siècle, en lien avec la réorientation des flux et l'insertion dans de nouveaux circuits commerciaux, tournés vers la France, après la décennie 1490. Ce mouvement renforce incontestablement les métairies situées dans les zones de marches, là où la croissance tout au long du Xv siècle a été aussi la plus vigoureuse.

Les $\mathrm{XIV}^{\mathrm{e}}$ et $\mathrm{XV}$ e siècles marquent ainsi un profond tournant dans l'histoire et la géographie des structures agraires du comté de Nantes. Les systèmes d'exploitation connaissent des rythmes et des évolutions contrastées, différentes selon leur localisation dans le comté. Au nord de la Loire, les censives résistent aux difficultés croissantes de la seconde moitié du XV siècle, même si elles en sortent affaiblies. La petite propriété paysanne est

temps des laboureurs. Travail, ordre social et croissance en Europe (XI'-XIV siècle), Paris, Albin Michel, 2012, p. 221-255).

91. Extrait de l'aveu rendu le 17 janvier 1454 (n. st.) par Olivier Gladonnet (Arch. dép. de Loire-Atlantique, B 1875 (paroisse des Touches).

92. Extrait de l'aveu rendu le 22 février 1393 (n. st.) par Jahen de Cleuz (Arch. dép. de Loire-Atlantique, B 1482 (paroisse de Saint-Nazaire). 
une réalité bien vivante dans ces campagnes, en lien avec les sieuries, fort nombreuses. Les territoires du sud et de l'est présentent d'autres traits, fortement inspirés par les influences extérieures, mais aussi par des structures économiques plus variées. Le renforcement du binôme manoir-métairie(s) y est beaucoup plus net et s'accentue encore avec la reconstruction agraire qui débute timidement à la fin du $\mathrm{Xv}^{\mathrm{e}}$ siècle. Un encadrement seigneurial plus étroit, formé par les châtellenies et les baronnies, oriente ces évolutions. Les échanges commerciaux avec la France ou le reste de la Bretagne accompagnent en outre la réorientation vers les métairies, plus tournées vers les marchés extérieurs que les censives. Se mettent donc en place des structures de longue durée, qui contribuent à définir de nouveaux rapports entre seigneurs/sieurs et exploitation de la terre.

\section{RÉSUMÉ}

Les $\mathrm{XIV}^{\mathrm{e}}$ et $\mathrm{XV}^{\mathrm{e}}$ siècles marquent un tournant dans l'organisation spatiale des campagnes nantaises. Face aux multiples bouleversements (guerres, développement et réorientation des échanges extérieurs, reconstruction des campagnes à la fin du Moyen Âge), les seigneurs ruraux modifient, de manière lente et progressive, les structures agraires en accordant une place grandissante aux redevances partiaires. Les métairies connaissent alors une période d'expansion sans toutefois supplanter totalement les censives, qui continuent de dominer les paysages agraires du comté de Nantes. Cet article se propose de retracer dans leurs grandes lignes les principales évolutions qui ont conduit à ce développement du métayage, en croisant démarche historique et approche multiscalaire.

\section{ABSTRACT}

The fourteenth and fifteenth centuries marked a turning point in the spatial organisation of the Nantes countryside. Facing numerous and profound changes (war, the development and reorganisation of external exchanges, the reconstruction of the countryside at the end of the Middle Ages), rural lords slowly and progressively modified agrarian structures to allow a larger role to metayage. As a result there was a rise in the number of such farms though they never totally replaced quit-rent tenants that continued to dominate in the county of Nantes. This article seeks to retrace the main steps that led to the development of metayage by using both historical and multiscalar approaches. 